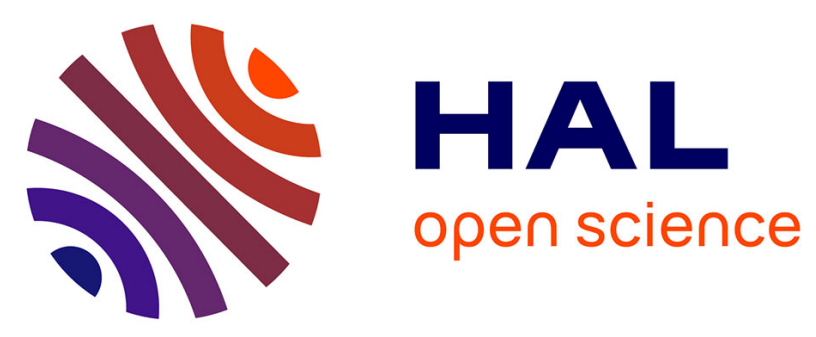

\title{
Speckle observations with PISCO in Merate (Italy): $\mathrm{XV}$. Astrometric measurements of visual binaries in 2014, and new orbits for ADS 671, 1615, 1709, 5447, 10075 and 12447.
}

Jean-Louis Prieur, M. Scardia, L. Pansecchi, R. Argyle, A. Zanutta, E. Aristidi

\section{To cite this version:}

Jean-Louis Prieur, M. Scardia, L. Pansecchi, R. Argyle, A. Zanutta, et al.. Speckle observations with PISCO in Merate (Italy): XV. Astrometric measurements of visual binaries in 2014, and new orbits for ADS 671, 1615, 1709, 5447, 10075 and 12447.. Astronomical Notes / Astronomische Nachrichten, 2017, 338 (1), pp.74-90. 10.1002/asna.201613244 . hal-02357170

\author{
HAL Id: hal-02357170 \\ https://hal.science/hal-02357170
}

Submitted on 9 Nov 2019

HAL is a multi-disciplinary open access archive for the deposit and dissemination of scientific research documents, whether they are published or not. The documents may come from teaching and research institutions in France or abroad, or from public or private research centers.
L'archive ouverte pluridisciplinaire HAL, est destinée au dépôt et à la diffusion de documents scientifiques de niveau recherche, publiés ou non, émanant des établissements d'enseignement et de recherche français ou étrangers, des laboratoires publics ou privés. 


\title{
Speckle observations with PISCO in Merate (Italy): XV. Astrometric measurements of visual binaries in 2014, and new orbits for ADS 671, 1615, 1709, 5447, 10075 and 12447.
}

\author{
J.-L. Prieur ${ }^{1,2}$, M. Scardia ${ }^{3}$, L. Pansecchi ${ }^{3}$, R.W. Argyle ${ }^{4}$, A. Zanutta ${ }^{3}$, and E. Aristidi ${ }^{5}$ \\ 1 Université de Toulouse - UPS-OMP - IRAP, Toulouse, France \\ 2 CNRS - IRAP, 14 avenue Edouard Belin, 31400 Toulouse, France \\ 3 INAF - Osservatorio Astronomico di Brera, Via E. Bianchi 46, 23807 Merate, Italy \\ 4 Institute of Astronomy, Madingley Road, Cambridge, CB3 0HA, United Kingdom \\ ${ }^{5}$ Université de Nice-Sophia Antipolis -Laboratoire Lagrange-CNRS-OCA, Parc Valrose 06108 Nice cedex 2, \\ France
}

Received January 17, 2017; accepted

\begin{abstract}
Key words Stars: binaries: close - binaries: visual — astrometry — techniques: interferometric — stars: individual (ADS 671, ADS 1615, ADS 1709, ADS 5447, ADS 10075 and ADS 12447)

We present relative astrometric measurements of visual binaries, made in 2014 with the speckle camera PISCO at the 102-cm Zeiss telescope of Brera Astronomical Observatory, in Merate. Our observing list contains orbital couples as well as binaries whose motion is still uncertain. We obtained 224 new measurements of 218 visual binary stars, with angular separations in the range $0^{\prime \prime} .15-10^{\prime \prime}$, and an average accuracy of $0^{\prime \prime} .015$. The mean error on the position angles is $0^{\circ} .5$. Most of the position angles were determined without the usual $180^{\circ}$ ambiguity with the application of triple-correlation techniques and/or by inspection of the long integration files. We complete this data with the results of a study of the multiple system ADS 6993 with PISCO during the period 2004-2014 and propose a new method to resolve part of the 180-degree ambiguity with the autocorrelations only. We then present new revised orbits for ADS 671, 1615, 1709, 5447, 10075 and 12447, partly derived from PISCO observations. The corresponding estimated values for the masses of those systems are compatible with the spectral types.
\end{abstract}

\section{Introduction}

This paper presents the results of speckle observations of visual binary stars made in Merate (Italy) in 2014 with the Pupil Interferometry Speckle camera and COronagraph (PISCO) on the 102-cm Zeiss telescope of INAF - Osservatorio Astronomico di Brera (OAB, Brera Astronomical Observatory). It is the fifteenth of a series whose purpose is to contribute to the determination of binary orbits (Scardia et al. 2005, 2006, 2007, 2008a, Prieur et al. 2008, Scardia et al. 2009, Prieur et al. 2009, Scardia et al. 2010, Prieur et al. 2010, Scardia et al. 2011, Prieur et al. 2012, Scardia et al. 2013, Prieur et al. 2014, and Scardia et al. 2015a, herein: Papers I to XIV). The focal instrument PISCO was developed at Observatoire Midi-Pyrénées (France) and first used at Pic du Midi from 1993 to 1998. It was moved to Merate in 2003 and installed on the INAF Zeiss telescope that has been dedicated to binary star observations since that epoch.

In Sect. 2, we briefly describe our observations. In Sect. 3, we present and discuss the astrometric measurements. We also compare those measurements with the ephemerides computed with the published orbital elements, when available. In Sect. 4, we describe the results of a study of the multiple system ADS 6993 with PISCO in Merate during the period 2004-2014. Finally in Sect. 5 we present the new revised orbits that we have computed for ADS 671, 1615, 1709, 5447, 10075 and 12447, and discuss the estimated values for the masses of those systems.

\section{Observations}

The observations were carried out with the PISCO speckle camera and the ICCD (Intensified Charge Coupled Device) detector belonging to Nice University (France). This instrumentation is presented in Prieur et al. (1998) and our observing procedure is described in detail in Paper VI.

Our observing list basically includes all the visual binaries for which new measurements are needed to improve their orbits, that are accessible with our instrumentation. It consists of a few thousands objects. A detailed description can be found in our previous papers (e.g., Paper VI).

The distribution of the angular separations measured in this paper is displayed in Fig. 1a and shows 

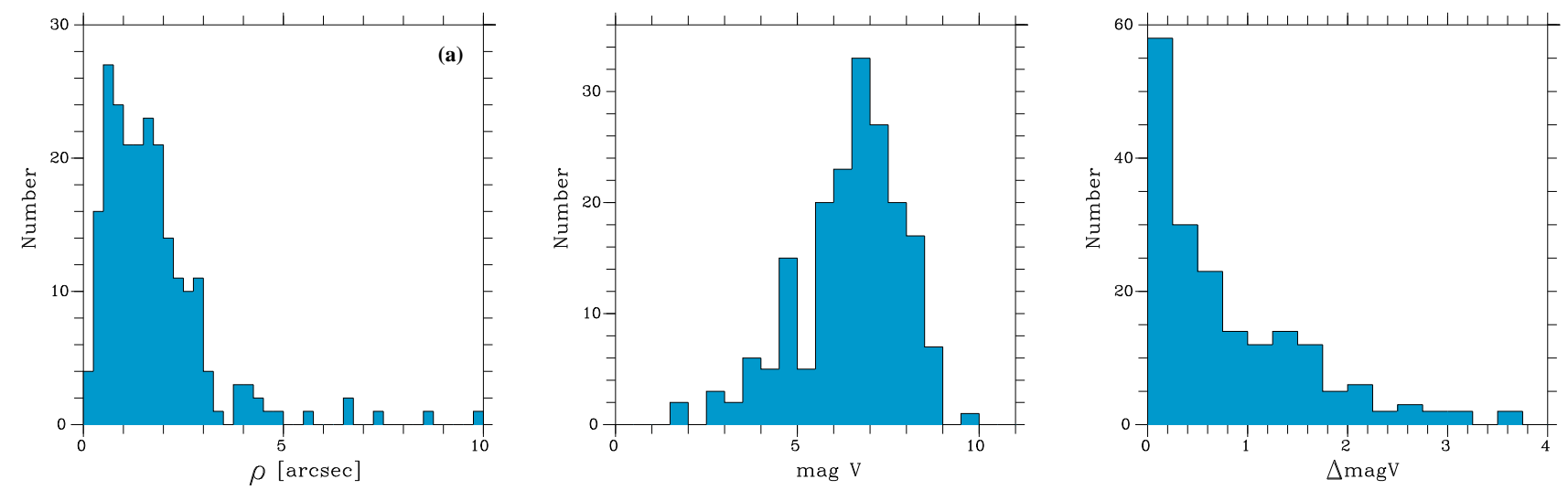

Fig. 1 Distribution of the angular separations of the 224 measurements of Table 1 (a), the total visual magnitudes of the corresponding binaries (b) and the differences of magnitude between their two components (c).

a maximum for $\rho \approx 0^{\prime \prime} .6$. The largest separation of $8^{\prime \prime} .72$ was obtained for STF 948 AC (ADS 5400). The smallest separations were measured for ADS 8804 and ADS 5447, with $\rho=0^{\prime \prime} .15$ and $\rho=0^{\prime \prime} .18$, respectively. Let us recall that the diffraction limit is $\rho_{d}=\lambda / D \approx$ $0^{\prime \prime} .13$ for the Zeiss telescope (aperture $D=1.02 \mathrm{~m}$ ) and the $R$ filter $(\lambda=650 \mathrm{~nm})$.

The distribution of the apparent magnitudes $m_{V}$ and of the difference of magnitudes $\Delta m_{V}$ between the two components are plotted in Figs. 1b and 1c, respectively. The telescope aperture and detector sensitivity led to a limiting magnitude of $m_{V}=10$ (Fig. 1b) and a maximum $\Delta m_{V}$ for speckle measurements of about 4.0 (Fig. 1c).

Using the Hipparcos parallaxes from ESA (1995), we have been able to construct the HR diagram of those binaries, which is displayed in Fig. 2. We only plotted the 183 objects for which the relative uncertainty on the parallax was smaller than $50 \%$.

\section{Astrometric measurements}

The 224 astrometric measurements obtained with the observations made in 2014 are displayed in Table 1. They concern 218 visual binaries. For each object, we report its WDS name (Washington Double Star Catalog, Mason et al. 2016, hereafter WDS catalog) in Col. 1, the official double star designation in Col. 2 (sequence is "discoverer-number"), and the ADS number in Col. 3 (Aitken, 1932) when available. For each observation, we then give the epoch in Besselian years (Col. 4), the filter (Col. 5), the focal length of the eyepiece used for magnifying the image (Col. 6), the angular separation $\rho$ (Col. 7) with its error (Col. 8) in arcseconds, and the position angle $\theta$ (Col. 9) with its error (Col. 10) in degrees. In Col. 11, we report some notes and some information about the secondary peaks of the autocorrelation files (e.g. diffuse, faint or elongated) or about the power spectrum (NF: no fringes).

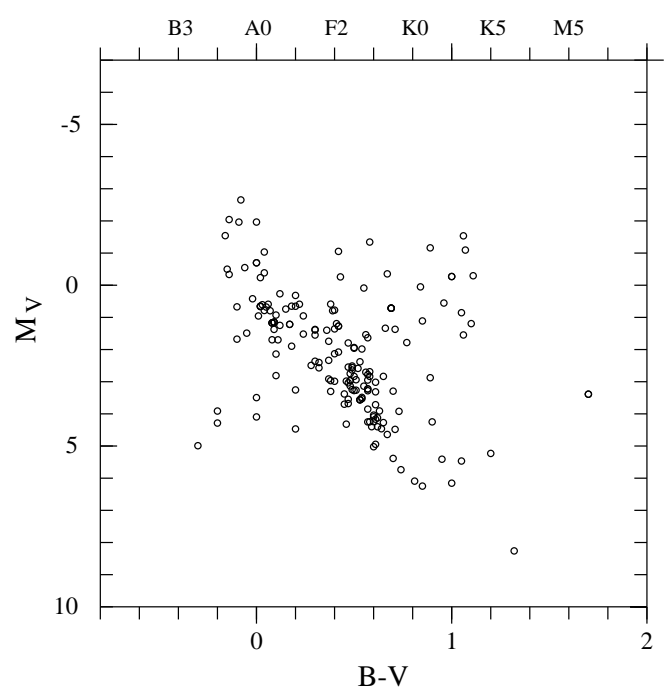

Fig. 2 HR diagram of the binaries measured in Table 1, for which Hipparcos parallaxes were obtained with a relative error smaller than $50 \%$ (i.e., 183 objects).

For the systems with a known orbit, the $(O-C)$ (Observed minus Computed) residuals of the $\rho$ and $\theta$ measurements are displayed in Cols. 13 and 14, respectively. The corresponding authors are given in Col. 12, using the bibliographic style of the "Sixth Catalog of Orbits of Visual Binary Stars" (Hartkopf \& Mason, 2016, hereafter OC6).

When not explicitly specified, the measurements refer to the $\mathrm{AB}$ components of those systems. In Col. 14, the symbol $Q$ indicates that there was a quadrant inconsistency between our measures and the positions derived from the orbital elements published for this object.

The characteristics of the $\mathrm{R}$ and RL filters used for obtaining those measurements are given in Table 1 of Paper XII. Some objects were observed without any filter because they were too faint. This is indicated with W (for "white" light) in the filter column (Col. 5 of 
Table 1 Table of speckle measurements and O-C residuals with published orbits (begin.)

\begin{tabular}{|c|c|c|c|c|c|c|c|c|c|c|c|c|c|}
\hline WDS & Name & ADS & Epoch & Fil. & $\begin{array}{l}\text { Eyep. } \\
(\mathrm{mm})\end{array}$ & $\begin{array}{c}\rho \\
\left({ }^{\prime \prime}\right)\end{array}$ & $\begin{array}{l}\sigma_{\rho} \\
\left({ }^{\prime \prime}\right)\end{array}$ & $\begin{array}{c}\theta \\
\left({ }^{\circ}\right)\end{array}$ & $\begin{array}{l}\sigma_{\theta} \\
\left(^{\circ}\right)\end{array}$ & Notes & Orbit & $\begin{array}{r}\Delta \rho(\mathrm{O}-\mathrm{C}) \\
\left({ }^{\prime \prime}\right)\end{array}$ & $\begin{array}{r}\Delta \theta(\mathrm{O}-\mathrm{C}) \\
\left({ }^{\circ}\right)\end{array}$ \\
\hline $00134+2659$ & STT2 & 161 & 2014.042 & $\mathrm{R}$ & 20 & 0.419 & 0.013 & $157.2^{*}$ & ${ }^{*} 1.0$ & & Hrt2008 & 0.01 & 0.9 \\
\hline $00210+6740$ & HJ1018 & 283 & 2014.042 & $\mathrm{R}$ & 32 & 1.691 & 0.015 & $86.2^{*}$ & * 0.7 & & Sod1999 & -0.08 & -1.5 \\
\hline $00214+6700$ & STT6AB & 293 & 2014.042 & $\mathrm{R}$ & 20 & 0.660 & 0.017 & $153.4^{*}$ & * 1.2 & $\mathrm{NF}$ & Sca2000b & -0.04 & -0.8 \\
\hline $02020+0246$ & STF202 & 1615 & 2014.042 & $\mathrm{R}$ & 20 & 1.812 & 0.007 & $263.9^{*}$ & * 0.3 & & Sca1983f & 0.05 & 2.2 \\
\hline$"$ & $"$ & $"$ & . & $"$ & $"$ & $"$ & $"$ & - & $"$ & & This paper & -0.02 & 0.0 \\
\hline $02037+2556$ & STF208AB & 1631 & 2014.042 & $\mathrm{R}$ & 20 & 1.333 & 0.007 & $342.4^{*}$ & * 0.3 & & Pop1969b & 0.04 & -3.2 \\
\hline $02062+2507$ & STF212 & 1654 & 2014.042 & $\mathrm{R}$ & 32 & 2.000 & 0.053 & $160.8^{*}$ & * 0.4 & & & & \\
\hline $03122+3713$ & STF360 & 2390 & 2014.146 & $\mathrm{R}$ & 32 & 2.883 & 0.035 & $124.2^{*}$ & * 0.3 & Diffuse & WSI2004a & 0.03 & -1.6 \\
\hline $03344+2428$ & STF412AB & 2616 & 2014.042 & $\mathrm{R}$ & 20 & 0.724 & 0.010 & 352.8 & 0.6 & & Sca2002a & -0.02 & 0.8 \\
\hline & & & 2014.146 & $\mathrm{R}$ & 20 & 0.724 & 0.008 & 351.8 & 0.6 & Diffuse & Sca2002a & -0.02 & -0.2 \\
\hline $04301+1538$ & STF554 & 3264 & 2014.198 & $\mathrm{R}$ & 20 & 1.526 & 0.008 & $16.0^{*}$ & * 0.7 & Diffuse & Baz1980a & -0.07 & 0.7 \\
\hline $04433+5931$ & A1013 & 3391 & 2014.201 & $\mathrm{R}$ & 10 & 0.391 & 0.003 & 296.9 & 0.9 & & Doc1990c & -0.01 & -1.1 \\
\hline $05135+0158$ & STT517AB & 3799 & 2014.198 & $\mathrm{R}$ & 20 & 0.722 & 0.010 & 239.7 & 0.5 & & Tok2014a & 0.04 & -0.7 \\
\hline $05148+1232$ & HU1224 & 3822 & 2014.198 & $\mathrm{R}$ & 32 & 1.071 & 0.017 & $114.3^{*}$ & * 0.8 & & Hrt2011a & 0.04 & 0.3 \\
\hline $05308+0557$ & STF728 & 4115 & 2014.198 & $\mathrm{R}$ & 20 & 1.289 & 0.013 & $45.1^{*}$ & * 0.4 & & USN1999b & -0.02 & 0.6 \\
\hline $05352+3358$ & AG97 & 4165 & 2014.201 & $\mathrm{R}$ & 32 & 2.091 & 0.017 & $269.9^{*}$ & * 0.8 & & & & \\
\hline $05355-0422$ & STF750 & 4192 & 2014.201 & $\mathrm{R}$ & 32 & 4.344 & 0.022 & $59.5^{*}$ & * 0.3 & & & & \\
\hline $05371+2655$ & STF749AB & 4208 & 2014.198 & $\mathrm{R}$ & 20 & 1.172 & 0.008 & $139.9^{*}$ & * 0.5 & & Sca2007a & 0.00 & $-0.3^{Q}$ \\
\hline $06221+5922$ & STF881Aa-B & 4950 & 2014.198 & $\mathrm{R}$ & 20 & 0.638 & 0.028 & $149.1^{*}$ & * 0.7 & & Zirm2013 & 0.00 & 1.8 \\
\hline $06275+2432$ & AG112AB & 5071 & 2014.198 & $\mathrm{R}$ & 32 & 2.903 & 0.039 & 208.6 & 1.8 & & & & \\
\hline $06404+4058$ & STF945 & 5296 & 2014.264 & $\mathrm{R}$ & 20 & 0.472 & 0.010 & $334.4^{*}$ & * 1.1 & & Nov2007d & -0.00 & -1.5 \\
\hline $06425+6612$ & MLR318 & - & 2014 & $\mathrm{R}$ & 20 & 1.685 & 0.008 & $308.8^{*}$ & * 0.4 & & Pal2005b & -0.01 & 0.1 \\
\hline $06462+5927$ & STF948AB & 5400 & 2014.196 & $\mathrm{R}$ & 20 & 1.906 & 0.010 & $67.4^{*}$ & * 0.3 & & WSI2006b & 0.01 & 0.2 \\
\hline $06462+5927$ & STF948AC & 5400 & 2014.196 & $\mathrm{R}$ & 32 & 8.722 & 0.044 & $308.3^{*}$ & $* 0.3$ & & & & \\
\hline $06474+1812$ & STT156 & 5447 & 2014.239 & $\mathrm{R}$ & 10 & 0.184 & 0.004 & 143.7 & 1.7 & & Sca2005a & -0.04 & -12.6 \\
\hline & $"$ & & 然 & $"$ & $"$ & 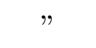 & $"$ & & $\because$ & & This paper & 0.01 & -1.9 \\
\hline $06487+0737$ & $\mathrm{~A} 2731$ & 5469 & 2014.239 & $\mathrm{R}$ & 32 & 1.363 & 0.027 & $64.8^{*}$ & * 0.6 & & Pru2012 & -0.01 & -1.4 \\
\hline $06531+5927$ & STF963 & 5514 & 2014.239 & $\mathrm{R}$ & 10 & 0.259 & 0.004 & $348.1^{*}$ & * 0.7 & & Sca2008d & -0.01 & 1.4 \\
\hline $06546+1311$ & STF982 & 5559 & 2014.239 & $\mathrm{R}$ & 32 & 7.394 & 0.042 & $143.0^{*}$ & * 0.4 & & Msn2014b & 0.09 & -0.1 \\
\hline $06555+3010$ & STF981 & 5570 & 2014.196 & $\mathrm{R}$ & 32 & 0.877 & 0.027 & $120.8^{*}$ & * 1.3 & & Hop1971 & -0.11 & $6.6^{Q}$ \\
\hline $06573+5825$ & STT159 & 5586 & 2014.239 & $\mathrm{R}$ & 10 & 0.674 & 0.003 & $232.9^{*}$ & * 0.3 & & Sod1999 & 0.02 & 1.4 \\
\hline $07128+2713$ & STF1037 & 5871 & 2014.196 & $\mathrm{R}$ & 20 & 0.943 & 0.008 & $305.9^{*}$ & * 0.4 & & Sca2015 & -0.01 & -0.6 \\
\hline $07176+0918$ & STT170 & 5958 & 2014.201 & $\mathrm{R}$ & 10 & 0.389 & 0.004 & 303.7 & 0.3 & Elongated & Doc2007e & 0.01 & -7.8 \\
\hline $07303+4959$ & STF1093 & 6117 & 2014.198 & $\mathrm{R}$ & 20 & 0.899 & 0.012 & $204.4^{*}$ & * 1.2 & & Hrt2009 & 0.02 & 0.5 \\
\hline $07345+1218$ & STF1116 & 6180 & 2014.201 & $\mathrm{R}$ & 32 & 1.697 & 0.017 & $96.1^{*}$ & * 0.5 & & & & \\
\hline $07346+3153$ & STF1110 & 6175 & 2014.196 & $\mathrm{R}$ & 20 & 4.966 & 0.025 & $55.1^{*}$ & * 0.3 & Elongated & Doc $2014 \mathrm{~g}$ & -0.02 & -0.1 \\
\hline $07401+0514$ & STF1126 & 6263 & 2014.198 & $\mathrm{R}$ & 20 & 0.859 & 0.010 & $174.6^{*}$ & * 0.6 & & Zir2015a & 0.02 & -1.5 \\
\hline $07417+3726$ & STT177 & 6276 & 2014.245 & $\mathrm{R}$ & 20 & 0.529 & 0.008 & 150.1 & 1.9 & & Hei1982c & -0.01 & 4.9 \\
\hline $07486+2308$ & WRH15 & 378 & 2014.201 & $\mathrm{R}$ & 10 & 0.259 & 0.003 & 31.6 & 1.9 & & 002 & -0.00 & 2.6 \\
\hline $07573+0108$ & STT185 & 6483 & 2014.239 & $\mathrm{R}$ & 20 & 0.345 & 0.012 & $20.8^{*}$ & * 1.2 & & Doc1994d & -0.05 & 1.7 \\
\hline $08024+0409$ & STF1175 & 6532 & 2014.239 & $\mathrm{R}$ & 32 & 1.366 & 0.033 & $284.9^{*}$ & * 0.8 & & Ole2001 & -0.02 & -5.3 \\
\hline $08041+3302$ & STT187 & 6549 & 2014.201 & $\mathrm{R}$ & 10 & 0.424 & 0.004 & 337.4 & 0.3 & & Msn1999a & 0.01 & -0.7 \\
\hline $08095+3213$ & STF1187 & 6623 & 2014.239 & $\mathrm{R}$ & 32 & 3.072 & 0.017 & $21.4^{*}$ & * 0.3 & & Ole2001 & 0.12 & 0.9 \\
\hline $08122+1739$ & STF1196AB & 6650 & 2014.198 & $\mathrm{R}$ & 20 & 1.120 & 0.008 & $24.5^{*}$ & * 0.3 & & WSI2006b & 0.01 & 1.6 \\
\hline $08122+1739$ & STF1196AC & 6650 & 2014.198 & $\mathrm{R}$ & 32 & 6.567 & 0.042 & $62.3^{*}$ & * 0.5 & & & & \\
\hline $08198+0357$ & FIN346 & - & 2014.245 & $\mathrm{R}$ & 10 & 0.229 & 0.003 & 54.6 & 1.6 & Elon & & & \\
\hline $08213-0136$ & STF1216 & 6762 & 2014.245 & $\mathrm{R}$ & 10 & 0.526 & 0.006 & 306.4 & 0.3 & Elongated & Tok2 & 0.00 & -0.8 \\
\hline $08468+0625$ & SP1AB & 6993 & 2014.201 & $\mathrm{R}$ & 10 & 0.261 & 0.003 & $194.0^{*}$ & * 0.5 & & Sod1999 & -0.01 & 0.2 \\
\hline $08468+0625$ & STF1273AB,C & 6993 & 2014.201 & $\mathrm{RL}$ & 20 & 2.787 & 0.014 & $305.2^{*}$ & $* 0.3$ & Elongated & Dru2014 & -0.03 & -1.2 \\
\hline $08468+0625$ & STF1273AC & 6993 & 2014.201 & $\mathrm{RL}$ & 20 & 2.769 & 0.014 & $304.5^{*}$ & * 0.3 & & & & \\
\hline $08531+5457$ & A 1584 & 7054 & 2014.196 & $\mathrm{R}$ & 20 & 0.651 & 0.012 & $89.4^{*}$ & * 0.5 & & Msn2014a & -0.02 & -1.2 \\
\hline $08542+3035$ & STF1291 & 7071 & 2014.201 & $\mathrm{R}$ & 20 & 1.507 & 0.008 & $308.9^{*}$ & * 0.3 & & & & \\
\hline $08554+7048$ & STF1280 & 7067 & 2014.239 & $\mathrm{R}$ & 32 & 3.005 & 0.022 & $354.9^{*}$ & $* 0.3$ & & Hei1997 & 0.10 & -0.7 \\
\hline $09012+0245$ & STF1302AB & 7141 & 2014.245 & $\mathrm{R}$ & 32 & 2.707 & 0.055 & 235.8 & 0.9 & & & & \\
\hline $09020+0240$ & BU211 & 7152 & 2014.245 & W & 32 & 2.170 & 0.082 & $269.2^{*}$ & * 0.6 & Diffuse & & & \\
\hline $09036+4709$ & A1585 & 7158 & 2014.196 & $\mathrm{R}$ & 10 & 0.272 & 0.005 & $288.8^{*}$ & * 0.8 & Elongated & Hrt2000a & -0.01 & 1.1 \\
\hline $09051+3931$ & AG160 & 7167 & 2014.264 & $\mathrm{R}$ & 32 & 3.987 & 0.020 & $59.3^{*}$ & * 0.7 & & & & \\
\hline
\end{tabular}


Table 1 Table of speckle measurements and O-C residuals with published orbits (cont.)

\begin{tabular}{|c|c|c|c|c|c|c|c|c|c|c|c|c|}
\hline WDS & Name & ADS & Epoch & Fil. & $\begin{array}{l}\text { Eyep. } \\
(\mathrm{mm})\end{array}$ & $\begin{array}{c}\rho \\
\left({ }^{\prime \prime}\right)\end{array}$ & $\begin{array}{l}\sigma_{\rho} \\
\left({ }^{\prime \prime}\right)\end{array}$ & $\begin{array}{cc}\theta & \sigma_{\theta} \\
\left(^{\circ}\right) & \left(^{\circ}\right)\end{array}$ & Notes & Orbit & $\begin{array}{r}\Delta \rho(\mathrm{O}-\mathrm{C}) \\
\left({ }^{\prime \prime}\right)\end{array}$ & $\begin{array}{r}\Delta \theta(\mathrm{O}-\mathrm{C}) \\
\left(^{\circ}\right)\end{array}$ \\
\hline $09071+3037$ & AG162 & 7183 & 2014.264 & $\mathrm{~W}$ & 32 & 4.121 & 0.040 & $106.0^{*} 0.6$ & & & & \\
\hline $09080+8102$ & STF1284 & 7163 & 2014.264 & $\mathrm{R}$ & 32 & 2.528 & 0.017 & $167.6^{*} 0.4$ & & & & \\
\hline $09095+0256$ & STT197 & 7215 & 2014.245 & $\mathrm{R}$ & 32 & 1.373 & 0.017 & $67.1^{*} 0.6$ & & & & \\
\hline $09103+5223$ & STF1312 & 7212 & 2014.264 & $\mathrm{R}$ & 32 & 4.686 & 0.033 & $147.8^{*} 0.3$ & & & & \\
\hline $09104+6708$ & STF1306 & 7203 & 2014.196 & $\mathrm{R}$ & 20 & 4.330 & 0.022 & $348.0^{*} 0.3$ & $\mathrm{NF}$ & Sca2015 & 0.02 & -0.2 \\
\hline $09149+0413$ & BU455 & 7257 & 2014.245 & W & 20 & 1.770 & 0.029 & $69.1^{*} 0.5$ & & & & \\
\hline $09184+3522$ & STF1333 & 7286 & 2014.201 & $\mathrm{R}$ & 20 & 1.929 & 0.010 & $49.7^{*} 0.3$ & & & & \\
\hline $09210+3811$ & STF1338AB & 7307 & 2014.196 & $\mathrm{R}$ & 20 & 1.118 & 0.008 & $306.6^{*} 0.4$ & & Sca2002b & -0.03 & -0.3 \\
\hline $09235+3908$ & STF1344 & 7332 & 2014.269 & $\mathrm{~W}$ & 32 & 3.803 & 0.019 & $102.6^{*} 0.4$ & Diffuse & & & \\
\hline $09273+0614$ & STF1355 & 7380 & 2014.240 & $\mathrm{R}$ & 32 & 1.811 & 0.017 & $354.0^{*} 0.3$ & & Lin2011b & -0.01 & -0.4 \\
\hline $09285+0903$ & STF1356 & 7390 & 2014.264 & $\mathrm{R}$ & 20 & 0.785 & 0.010 & $107.7^{*} 0.7$ & & Mut2010b & -0.02 & -0.8 \\
\hline $09435+0612$ & A 2761 & 7499 & 2014.264 & $\mathrm{R}$ & 32 & 1.023 & 0.020 & $251.0 \quad 1.4$ & & & & \\
\hline $09521+5404$ & STT208 & 7545 & 2014.196 & $\mathrm{R}$ & 10 & 0.396 & 0.003 & $301.9^{*} 0.3$ & & Hei1996c & -0.02 & -1.3 \\
\hline $09524+2659$ & STF1389 & 7551 & 2014.264 & $\mathrm{R}$ & 32 & 2.452 & 0.017 & $288.3^{*} 0.4$ & & & & \\
\hline $10163+1744$ & STT215 & 7704 & 2014.245 & $\mathrm{R}$ & 20 & 1.480 & 0.008 & $176.6^{*} 0.3$ & & Zae1984 & -0.07 & -2.0 \\
\hline $10269+1713$ & STT217 & 7775 & 2014.313 & $\mathrm{R}$ & 20 & 0.771 & 0.012 & $148.5^{*} 0.5$ & Diffuse & Sca2013b & -0.01 & 0.1 \\
\hline $10556+2445$ & STF1487 & 7979 & 2014.395 & $\mathrm{R}$ & 32 & 6.609 & 0.033 & $112.2^{*} 0.3$ & & & & \\
\hline $11037+6145$ & BU1077 & 8035 & 2014.395 & $\mathrm{R}$ & 10 & 0.714 & 0.004 & $355.4^{*} 0.4$ & & Sca2011a & -0.01 & -1.1 \\
\hline $11182+3132$ & STF1523AB & 8119 & 2014.264 & $\mathrm{R}$ & 20 & 1.728 & 0.009 & $180.1^{*} 0.3$ & & Hei1996b & 0.00 & -0.4 \\
\hline $11190+1416$ & STF1527 & 8128 & 2014.264 & $\mathrm{R}$ & 10 & 0.286 & 0.005 & $237.2^{*} 0.3$ & & Tok2012b & -0.01 & 0.7 \\
\hline $11239+1032$ & STF1536 & 8148 & 2014.264 & $\mathrm{R}$ & 20 & 2.066 & 0.010 & $96.9^{*} 0.3$ & & Sod1999 & -0.02 & 0.4 \\
\hline $11363+2747$ & STF 1555 & 8231 & 2014.264 & $\mathrm{R}$ & 20 & 0.720 & 0.008 & $148.8^{*} 0.3$ & & Doc2007i & 0.04 & -1.3 \\
\hline $11486+1417$ & BU603 & 8311 & 2014.396 & $\mathrm{R}$ & 20 & 0.981 & 0.008 & $331.5^{*} 0.5$ & & USN2006b & -0.10 & -1.6 \\
\hline $11561+4533$ & STF1581 & 8354 & 2014.396 & W & 32 & 2.424 & 0.017 & $170.4^{*} 0.7$ & & & & \\
\hline $12417-0127$ & STF1670 & 8630 & 2014.420 & $\mathrm{R}$ & 20 & 2.149 & 0.011 & $\begin{array}{ll}7.2 & 0.3\end{array}$ & & Sca2007c & -0.03 & -0.1 \\
\hline $12564-0057$ & STT256 & 8708 & 2014.428 & $\mathrm{R}$ & 32 & 1.056 & 0.022 & $99.4^{*} 1.5$ & & Zir2015a & -0.00 & -0.9 \\
\hline 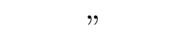 & $"$ & $"$ & 2014.420 & $\mathrm{R}$ & 32 & 1.024 & 0.022 & $100.3^{*} 1.0$ & & Zir2015a & -0.04 & 0.0 \\
\hline $13007+5622$ & BU1082 & 8739 & 2014.396 & $\mathrm{R}$ & 20 & 0.879 & 0.009 & $113.8^{*} 0.4$ & & Sca2012c & -0.05 & 0.5 \\
\hline $13100+1732$ & STF1728 & 8804 & 2014.396 & $\mathrm{R}$ & 10 & 0.149 & 0.003 & $\begin{array}{ll}17.0 & 0.8\end{array}$ & Uncertain & WSI2006b & -0.03 & 5.0 \\
\hline $13235+2914$ & HO260 & 8887 & 2014.396 & $\mathrm{R}$ & 32 & 1.602 & 0.027 & $87.4^{*} 1.2$ & & Zir2013a & -0.02 & 0.4 \\
\hline $13284+1543$ & STT266 & 8914 & 2014.396 & $\mathrm{R}$ & 32 & 1.972 & 0.017 & $357.1^{*} 0.4$ & & Hrt2011d & -0.03 & -0.3 \\
\hline $13288+5956$ & STF1752AB & 8919 & 2014.442 & W & 20 & 0.950 & 0.008 & $106.9^{*} 0.7$ & & & & \\
\hline $13329+3454$ & STT269 & 8939 & 2014.437 & $\mathrm{R}$ & 10 & 0.292 & 0.004 & 224.71 .1 & $\mathrm{NF}$ & Hei1997 & -0.02 & 0.0 \\
\hline $13329+4908$ & STF1758 & 8940 & 2014.437 & $\mathrm{R}$ & 32 & 3.351 & 0.018 & $291.5^{*} 0.3$ & & & & \\
\hline $13343-0019$ & STF1757 & 8949 & 2014.442 & $\mathrm{R}$ & 32 & 1.756 & 0.027 & $138.5^{*} 0.3$ & & Hei1988d & -0.00 & 0.3 \\
\hline $13356+4939$ & AG190 & 8964 & 2014.442 & W & 32 & 2.625 & 0.017 & $12.9^{*} 0.6$ & Elongated & & & \\
\hline $13375+3618$ & STF1768 & 8974 & 2014.442 & $\mathrm{R}$ & 20 & 1.726 & 0.009 & $95.6^{*} 0.3$ & & Sod1999 & 0.03 & 0.4 \\
\hline $13377+5043$ & STF 1770 & 8979 & 2014.442 & $\mathrm{R}$ & 20 & 1.697 & 0.008 & $121.4^{*} 0.3$ & & & & \\
\hline $13577+5200$ & A1614 & 9071 & 2014.428 & $\mathrm{R}$ & 32 & 1.383 & 0.018 & $299.4^{*} 0.8$ & & RAO2015 & -0.01 & $-0.9^{Q}$ \\
\hline $14131+5520$ & STF1820 & 9167 & 2014.396 & $\mathrm{R}$ & 32 & 2.781 & 0.023 & $121.2^{*} 0.4$ & & Kiy1998 & 0.13 & -1.0 \\
\hline $14153+0308$ & STF1819 & 9182 & 2014.396 & $\mathrm{R}$ & 20 & 0.894 & 0.009 & $168.9^{*} 0.3$ & & Sca2012b & 0.00 & -0.1 \\
\hline $14203+4830$ & STF1834 & 9229 & 2014.437 & $\mathrm{R}$ & 20 & 1.641 & 0.008 & $101.9^{*} 0.3$ & & USN2000c & 0.06 & -1.8 \\
\hline $14270+0341$ & STF1842 & 9265 & 2014.437 & $\mathrm{R}$ & 32 & 2.737 & 0.027 & 198.10 .5 & & & & \\
\hline $14317+0150$ & AG195 & 9293 & 2014.437 & W & 32 & 1.810 & 0.017 & $\begin{array}{ll}337.1 & 0.3\end{array}$ & & & & \\
\hline $14369+4813$ & A 347 & 9324 & 2014.442 & W & 20 & 0.541 & 0.008 & $238.5 \quad 0.3$ & Elongated & Doc2004a & -0.03 & -1.1 \\
\hline $14380+5135$ & STF1863 & 9329 & 2014.442 & $\mathrm{R}$ & 20 & 0.656 & 0.008 & $60.7^{*} 1.0$ & & Zir2013a & 0.01 & 0.4 \\
\hline $14411+1344$ & STF1865 & 9343 & 2014.510 & $\mathrm{R}$ & 10 & 0.437 & 0.003 & $291.7^{*} 0.3$ & Elongated & Sca2007f & -0.01 & 0.6 \\
\hline $14417+0932$ & STF1866 & 9345 & 2014.510 & $\mathrm{R}$ & 20 & 0.747 & 0.008 & 203.60 .5 & & & & \\
\hline $14428+0635$ & A1109AB & 9353 & 2014.538 & $\mathrm{RL}$ & 32 & 1.806 & 0.039 & $87.3^{*} 0.3$ & & WSI2006b & 0.08 & -1.1 \\
\hline $14463+0939$ & STF1879 & 9380 & 2014.437 & $\mathrm{R}$ & 32 & 1.714 & 0.018 & $82.4^{*} 0.3$ & & Msn1999a & -0.01 & -0.7 \\
\hline $14489+0557$ & STF1883 & 9392 & 2014.437 & $\mathrm{R}$ & 20 & 1.003 & 0.008 & $277.2 \quad 0.3$ & & USN2000c & 0.02 & 0.1 \\
\hline $14497+0759$ & A1110 & 9400 & 2014.538 & $\mathrm{R}$ & 20 & 0.661 & 0.009 & $244.6^{*} 0.9$ & & & & \\
\hline $14515+4456$ & STT287 & 9418 & 2014.437 & $\mathrm{R}$ & 20 & 0.658 & 0.008 & $2.1 \quad 1.2$ & & Hei1997 & -0.07 & 1.8 \\
\hline $14525+1844$ & BU31AB & 9423 & 2014.538 & $\mathrm{R}$ & 32 & 1.997 & 0.017 & $220.5^{*} 0.4$ & & Hrt2014a & -0.01 & -0.3 \\
\hline $14534+1542$ & STT288 & 9425 & 2014.543 & $\mathrm{R}$ & 20 & 1.017 & 0.008 & $158.9^{*} 0.3$ & & Hei1998 & -0.00 & 0.0 \\
\hline $15038+4739$ & STF1909 & 9494 & 2014.543 & $\mathrm{R}$ & 20 & 1.057 & 0.008 & $64.8^{*} 0.4$ & & Zir2011 & 0.01 & -1.4 \\
\hline $15056+1138$ & STF1907 & 9498 & 2014.543 & W & 20 & 0.858 & 0.008 & $347.8 \quad 0.6$ & Elongated & & & \\
\hline
\end{tabular}


Table 1 Table of speckle measurements and O-C residuals with published orbits (cont.)

\begin{tabular}{|c|c|c|c|c|c|c|c|c|c|c|c|c|}
\hline WDS & Name & ADS & Epoch & Fil. & $\begin{array}{l}\text { Eyep. } \\
\text { (mm) }\end{array}$ & $\begin{array}{c}\rho \\
\left({ }^{\prime \prime}\right)\end{array}$ & $\begin{array}{l}\sigma_{\rho} \\
\left({ }^{\prime \prime}\right)\end{array}$ & $\begin{array}{cc}\theta & \sigma_{\theta} \\
\left(^{\circ}\right) & \left(^{\circ}\right)\end{array}$ & Notes & Orbit & $\begin{array}{r}\Delta \rho(\mathrm{O}-\mathrm{C}) \\
\left({ }^{\prime \prime}\right)\end{array}$ & $\begin{array}{r}\Delta \theta(\mathrm{O}-\mathrm{C}) \\
\left({ }^{\circ}\right)\end{array}$ \\
\hline $15232+3017$ & STF1937AB & 9617 & 2014.511 & $\mathrm{R}$ & 10 & 0.651 & 0.003 & $202.4^{*} 0.4$ & Elongated & Sod1999 & -0.00 & 0.1 \\
\hline $15245+3723$ & STF1938BC & 9626 & 2014.511 & $\mathrm{R}$ & 20 & 2.256 & 0.011 & $4.4^{*} 0.3$ & & Sca2013a & 0.04 & 0.4 \\
\hline $15246+5413$ & HU149 & 9628 & 2014.511 & $\mathrm{R}$ & 20 & 0.673 & 0.008 & $270.2^{*} 0.7$ & & Zir2015a & 0.02 & -0.5 \\
\hline $15382+3615$ & HU1167AB & 9731 & 2014.582 & $\mathrm{R}$ & 32 & 1.358 & 0.023 & $77.2^{*} 1.9$ & $\mathrm{NF}$ & Dru1995 & 0.11 & -3.6 \\
\hline $15382+3615$ & STF1964CD & 9731 & 2014.582 & $\mathrm{R}$ & 32 & 1.585 & 0.028 & $20.2^{*} 0.4$ & & Dru1995 & 0.07 & 0.8 \\
\hline $15413+5959$ & STF1969 & 9756 & 2014.582 & $\mathrm{R}$ & 32 & 1.033 & 0.017 & $28.1^{*} 0.6$ & & RAO2015 & -0.01 & -0.6 \\
\hline $15589-0304$ & STF3101 & 9864 & 2014.538 & $\mathrm{R}$ & 32 & 2.226 & 0.017 & $73.3^{*} 0.6$ & & & & \\
\hline $16009+1316$ & STT303AB & 9880 & 2014.543 & $\mathrm{R}$ & 20 & 1.577 & 0.009 & $173.2^{*} 0.3$ & & Zir2015a & -0.01 & -0.2 \\
\hline $16115+1507$ & A1799 & 9952 & 2014.543 & $\mathrm{~W}$ & 20 & 0.783 & 0.009 & $\begin{array}{ll}116.4 & 0.7\end{array}$ & Elongated & Zir2014a & -0.00 & 0.7 \\
\hline $16289+1825$ & STF2052AB & 10075 & 2014.511 & $\mathrm{R}$ & 20 & 2.350 & 0.012 & $118.7^{*} 0.3$ & & Lmp2001a & 0.03 & 0.1 \\
\hline & & & & $"$ & $"$ & $"$ & $"$ & $" n$ & & This paper & -0.01 & -0.9 \\
\hline $16309+0159$ & STF2055AB & 10087 & 2014.511 & $\mathrm{R}$ & 20 & 1.387 & 0.008 & $40.7^{*} 0.4$ & & Hei1993b & -0.05 & 0.0 \\
\hline $16413+3136$ & STF2084 & 10157 & 2014.511 & $\mathrm{R}$ & 10 & 1.191 & 0.006 & $139.2^{*} 0.3$ & & Sod1999 & -0.01 & -2.3 \\
\hline $16433+2508$ & STF2089 & 10174 & 2014.582 & $\mathrm{~W}$ & 32 & 2.710 & 0.030 & $61.0^{*} 0.5$ & Faint & & & \\
\hline $16442+2331$ & STF2094AB & 10184 & 2014.582 & $\mathrm{R}$ & 20 & 1.150 & 0.011 & $\begin{array}{ll}72.3 & 0.3\end{array}$ & & & & \\
\hline $16448+3544$ & STF2097AB & 10193 & 2014.664 & $\mathrm{~W}$ & 32 & 1.826 & 0.017 & $79.8^{*} 0.7$ & & & & \\
\hline $16492+4559$ & BU627AB & 10227 & 2014.664 & $\mathrm{RL}$ & 20 & 2.102 & 0.011 & $42.5^{*} 0.3$ & & FMR2012a & -0.04 & 0.4 \\
\hline $16492+4559$ & BU627A,BC & 10227 & 2014.664 & $\mathrm{RL}$ & 20 & 2.021 & 0.010 & $38.9^{*} 0.3$ & & & & \\
\hline $16492+4559$ & BU627AC & 10227 & 2014.664 & $\mathrm{RL}$ & 20 & 1.941 & 0.010 & $35.3^{*} 0.3$ & & & & \\
\hline $16511+0924$ & STF2106 & 10229 & 2014.511 & $\mathrm{R}$ & 20 & 0.754 & 0.008 & $171.4^{*} 0.3$ & & Sca2001g & -0.04 & 0.1 \\
\hline $16514+0113$ & STT315 & 10230 & 2014.658 & $\mathrm{R}$ & 20 & 0.689 & 0.008 & $310.6^{*} 0.3$ & & Doc2007d & -0.03 & 0.2 \\
\hline $16518+2840$ & STF2107AB & 10235 & 2014.511 & $\mathrm{R}$ & 20 & 1.424 & 0.008 & $103.4^{*} 0.3$ & & Sca2003c & 0.03 & -1.3 \\
\hline $16564+6502$ & STF2118AB & 10279 & 2014.511 & $\mathrm{R}$ & 20 & 0.995 & 0.008 & $65.8^{*} 0.3$ & & Sca2002d & -0.15 & -0.9 \\
\hline $16588+0358$ & STF3107AB & 10285 & 2014.658 & $\mathrm{R}$ & 32 & 1.404 & 0.032 & $69.4^{*} 0.5$ & & & & \\
\hline $17020+0827$ & STF2114 & 10312 & 2014.582 & $\mathrm{R}$ & 20 & 1.341 & 0.008 & $195.5^{*} 0.5$ & & & & \\
\hline $17053+5428$ & STF2130AB & 10345 & 2014.582 & $\mathrm{~A}$ & 20 & 2.497 & 0.012 & $3.4^{*} 0.4$ & & Pru2012 & 0.01 & 0.1 \\
\hline $17082-0105$ & A1145 & 10355 & 2014.655 & $\mathrm{R}$ & 20 & 0.663 & 0.008 & $343.0^{*} 0.4$ & & WSI2006b & 0.00 & -0.0 \\
\hline $17178+4535$ & STF2152 & 10458 & 2014.672 & $\mathrm{~W}$ & 32 & 2.000 & 0.027 & $237.4^{*} 0.6$ & & & & \\
\hline $17179+4918$ & STF2153 & 10460 & 2014.672 & $\mathrm{~W}$ & 32 & 1.468 & 0.017 & $244.5^{*} 0.3$ & & & & \\
\hline $17237+3709$ & MCA $48 \mathrm{AB}$ & 10526 & 2014.656 & $\mathrm{R}$ & 20 & 4.054 & 0.020 & $320.0^{*} 0.3$ & & & & \\
\hline $17358+0100$ & STF2 & 10650 & 2014.658 & $\mathrm{R}$ & 32 & 2.993 & 0.018 & $78.1^{*} 0.3$ & & & & \\
\hline $17386+5546$ & STF2199 & 10699 & 2014.658 & $\mathrm{R}$ & 32 & 2.049 & 0.017 & $54.8^{*} 0.3$ & & Pop1995d & 0.12 & 2.0 \\
\hline $17403+6341$ & STF2218 & 10728 & 2014.664 & $\mathrm{R}$ & 32 & 1.458 & 0.017 & $309.6^{*} 0.3$ & & Zir2015a & 0.01 & -0.0 \\
\hline $17412+4139$ & STF2203 & 10722 & 2014.664 & $\mathrm{~W}$ & 20 & 0.759 & 0.008 & $291.3^{*} 0.3$ & Elongated & & & \\
\hline $17506+0714$ & STT337 & 10828 & 2014.658 & $\mathrm{~W}$ & 20 & 0.555 & 0.018 & $161.8^{*} 2.0$ & & Doc1990a & 0.03 & -1.6 \\
\hline $17520+1520$ & STT338AB & 10850 & 2014.582 & $\mathrm{R}$ & 20 & 0.822 & 0.008 & $163.7^{*} 0.8$ & & Pru2012 & -0.01 & -0.5 \\
\hline $17533+3605$ & STF2243 & 10874 & 2014.740 & $\mathrm{R}$ & 32 & 1.133 & 0.023 & $\begin{array}{ll}39.5 & 0.9\end{array}$ & & & & \\
\hline $17541+2949$ & AC9 & 10880 & 2014.735 & $\mathrm{R}$ & 32 & 1.155 & 0.017 & $240.7^{*} 0.7$ & & & & \\
\hline $17564+1820$ & STF2245AB & 10905 & 2014.735 & $\mathrm{R}$ & 20 & 2.635 & 0.017 & $111.3^{*} 0.3$ & Elongated & & & \\
\hline $17571+0004$ & STF2244 & 10912 & 2014.735 & $\mathrm{R}$ & 20 & 0.673 & 0.008 & $98.9^{*} 0.4$ & & Hei1997 & 0.15 & -2.5 \\
\hline $17571+4551$ & HU235 & 10934 & 2014.658 & $\mathrm{R}$ & 20 & 1.554 & 0.013 & $285.2^{*} 0.3$ & & & & \\
\hline $18065+4022$ & STF2282 & 11074 & 2014.656 & $\mathrm{R}$ & 32 & 2.657 & 0.017 & $81.5^{*} 0.3$ & & & & \\
\hline $18097+5024$ & HU674 & 11128 & 2014.740 & $\mathrm{R}$ & 20 & 0.756 & 0.018 & 215.40 .5 & & USN2002 & 0.25 & 5.6 \\
\hline $18101+1629$ & STF2289 & 11123 & 2014.656 & $\mathrm{R}$ & 20 & 1.229 & 0.008 & $218.3^{*} 0.3$ & & Hop1964b & -0.01 & 2.5 \\
\hline $18250-0135$ & $\mathrm{AC} 11$ & 11324 & 2014.754 & $\mathrm{R}$ & 20 & 0.852 & 0.008 & $354.3^{*} 0.3$ & & Hei1995 & 0.04 & -0.1 \\
\hline $18339+5221$ & A1377 & 11468 & 2014.754 & $\mathrm{R}$ & 10 & 0.236 & 0.005 & $131.1^{*} 1.1$ & & Pru2010 & -0.02 & 1.8 \\
\hline $18355+2336$ & STT359 & 11479 & 2014.825 & $\mathrm{R}$ & 20 & 0.736 & 0.012 & $4.8^{*} 0.9$ & & Sca2009a & -0.01 & 0.6 \\
\hline$"$ & $"$ & & 2014.828 & $\mathrm{R}$ & 20 & 0.742 & 0.008 & $4.9^{*} 0.7$ & & Sca2009a & -0.00 & 0.6 \\
\hline $18359+1659$ & STT358 & 11483 & 2014.825 & $\mathrm{R}$ & 20 & 1.643 & 0.008 & $148.4^{*} 0.3$ & Diffuse & Hei1995 & 0.13 & 1.9 \\
\hline $18374+7741$ & STT363 & 11584 & 2014.836 & $\mathrm{R}$ & 20 & 0.544 & 0.008 & $339.5^{*} 0.7$ & & Sca2009a & 0.03 & -1.9 \\
\hline $18389+5221$ & STF2368 & 11558 & 2014.836 & $\mathrm{R}$ & 20 & 1.888 & 0.009 & $139.9^{*} 0.3$ & & & & \\
\hline $18443+3940$ & STF2382AB & 11635 & 2014.820 & $\mathrm{R}$ & 20 & 2.263 & 0.011 & $346.1^{*} 0.3$ & Diffuse & Nov2006e & -0.01 & 0.1 \\
\hline 1 & & & 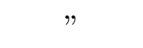 & $"$ & 然 & - & $"$ & $" \quad "$ & & WSI2004b & -0.08 & 0.1 \\
\hline $18443+3940$ & STF2383Cc-D & 11635 & 2014.820 & $\mathrm{R}$ & 20 & 2.382 & 0.012 & $76.2^{*} 0.3$ & Diffuse & & & \\
\hline $18455+0530$ & STF2375AB & 11640 & 2014.828 & $\mathrm{R}$ & 20 & 2.584 & 0.013 & $119.8^{*} 0.3$ & Diffuse & & & \\
\hline $18497+1041$ & STF2402 & 11722 & 2014.735 & $\mathrm{R}$ & 32 & 1.309 & 0.017 & $202.3^{*} 1.6$ & & & & \\
\hline $18520+1047$ & STF2408 & 11766 & 2014.828 & $\mathrm{~W}$ & 32 & 2.252 & 0.023 & $90.7^{*} 0.3$ & & & & \\
\hline
\end{tabular}


Table 1 Table of speckle measurements and O-C residuals with published orbits (cont.)

\begin{tabular}{|c|c|c|c|c|c|c|c|c|c|c|c|c|}
\hline WDS & Name & ADS & Epoch & Fil. & $\begin{array}{l}\text { Eyep. } \\
(\mathrm{mm})\end{array}$ & $\underset{\rho}{\rho}$ & $\begin{array}{l}\sigma_{\rho} \\
\left({ }^{\prime \prime}\right)\end{array}$ & $\begin{array}{cc}\theta & \sigma_{\theta} \\
\left(^{\circ}\right) & \left(^{\circ}\right)\end{array}$ & Notes & Orbit & $\begin{array}{r}\Delta \rho(\mathrm{O}-\mathrm{C}) \\
\left({ }^{\prime \prime}\right)\end{array}$ & $\begin{array}{r}\Delta \theta(\mathrm{O}-\mathrm{C}) \\
\left({ }^{\circ}\right)\end{array}$ \\
\hline $18526+1400$ & STF2412 & 11778 & 2014.828 & $\mathrm{~W}$ & 20 & 1.412 & 0.009 & $55.8^{*} 0.5$ & Elongated & & & \\
\hline $18594+2936$ & STF2430 & 11914 & 2014.740 & $\mathrm{R}$ & 32 & 1.527 & 0.017 & $\begin{array}{ll}186.8 & 0.6\end{array}$ & & & & \\
\hline $19143+1904$ & STF2484 & 12201 & 2014.830 & $\mathrm{R}$ & 32 & 2.159 & 0.020 & $240.0^{*} 0.3$ & & Hop1973b & -0.11 & 0.5 \\
\hline $19148+4756$ & A706 & 12229 & 2014.830 & $\mathrm{~W}$ & 20 & 1.555 & 0.009 & $73.8^{*} 0.3$ & Elongated & & & \\
\hline $19169+6312$ & STF2509 & 12296 & 2014.831 & $\mathrm{R}$ & 20 & 1.838 & 0.009 & $328.0^{*} 0.3$ & & Zir2014a & 0.00 & -0.4 \\
\hline $19252+3708$ & HJ1395 & 12427 & 2014.831 & W & 32 & 2.804 & 0.028 & $62.9^{*} 0.5$ & & & & \\
\hline $19266+2719$ & STF2525 & 12447 & 2014.740 & $\mathrm{R}$ & 32 & 2.181 & 0.023 & $289.2^{*} 0.5$ & & Hei1984b & 0.03 & -0.3 \\
\hline$"$ & $"$ & $"$ & $"$ & $"$ & $"$ & $"$ & $"$ & $" \quad "$ & & This paper & 0.02 & -0.3 \\
\hline$"$ & $"$ & $"$ & 2014.869 & $\mathrm{R}$ & 32 & 2.215 & 0.017 & $289.0^{*} 0.5$ & Diffuse & Hei1984b & 0.06 & -0.5 \\
\hline$"$ & $"$ & $"$ & $"$ & $"$ & $"$ & $"$ & $"$ & $"$, & & This paper & 0.05 & -0.5 \\
\hline $19420+4015$ & KUI94 & - & 2014.836 & $\mathrm{RL}$ & 10 & 0.467 & 0.003 & $157.9^{*} 0.3$ & & Hrt1996a & 0.01 & 0.5 \\
\hline $19429+4043$ & STT383 & 12831 & 2014.825 & $\mathrm{R}$ & 20 & 0.783 & 0.011 & $14.7^{*} 1.1$ & & & & \\
\hline $19438+3819$ & STT384 & 12851 & 2014.740 & $\mathrm{R}$ & 32 & 1.045 & 0.018 & $196.6^{*} 1.0$ & & & & \\
\hline $19450+4508$ & STF2579 & 12880 & 2014.740 & $\mathrm{RL}$ & 20 & 2.701 & 0.014 & $216.6^{*} 0.3$ & & Sca2012c & -0.02 & -0.9 \\
\hline & $"$ & & 2014.836 & $\mathrm{RL}$ & 20 & 2.712 & 0.014 & $216.6^{*} 0.3$ & & Sca2012c & -0.01 & -0.8 \\
\hline $19464+3344$ & STF2576FG & 12889 & 2014.740 & $\mathrm{R}$ & 32 & 3.018 & 0.017 & $156.8^{*} 0.3$ & & Sca1981f & -0.02 & -0.9 \\
\hline $19484+2212$ & STF2584 & 12957 & 2014.825 & $\mathrm{~W}$ & 20 & 1.902 & 0.014 & 293.10 .3 & Elongated & & & \\
\hline $19487+1149$ & STF2583 & 12962 & 2014.741 & $\mathrm{R}$ & 20 & 1.432 & 0.008 & $105.0^{*} 0.3$ & & & & \\
\hline $19540+1518$ & STF2596 & 13082 & 2014.798 & $\mathrm{R}$ & 32 & 2.072 & 0.018 & $296.5^{*} 0.5$ & & & & \\
\hline $19556+5226$ & STF2605 & 13148 & 2014.828 & $\mathrm{R}$ & 20 & 2.840 & 0.014 & $175.2^{*} 0.3$ & & & & \\
\hline $19584-0214$ & $\mathrm{AC} 12$ & 13178 & 2014.893 & $\mathrm{R}$ & 32 & 1.535 & 0.018 & $296.3^{*} 0.3$ & & & & \\
\hline $19586+3806$ & STF2609 & 13198 & 2014.798 & $\mathrm{R}$ & 32 & 1.907 & 0.018 & $22.3^{*} 0.3$ & & & & \\
\hline $20011+4816$ & STF2619AB & 13269 & 2014.893 & $\mathrm{R}$ & 32 & 4.187 & 0.037 & $238.7 \quad 0.3$ & & & & \\
\hline $20020+2456$ & STT395 & 13277 & 2014.896 & $\mathrm{R}$ & 20 & 0.769 & 0.008 & $126.0^{*} 0.4$ & & Zir2013a & -0.08 & -0.4 \\
\hline $20035+3601$ & STF2624Aa-B & 13312 & 2014.798 & $\mathrm{R}$ & 32 & 1.938 & 0.017 & $173.8^{*} 1.1$ & & & & \\
\hline $20042+1148$ & STF2620 & 13320 & 2014.893 & $\mathrm{R}$ & 32 & 1.887 & 0.030 & $284.9^{*} 0.4$ & & & & \\
\hline $20184+5524$ & STF2671 & 13692 & 2014.798 & $\mathrm{R}$ & 32 & 3.772 & 0.022 & $336.9^{*} 0.6$ & & & & \\
\hline $20248+3545$ & BU432 & 13830 & 2014.882 & $\mathrm{R}$ & 32 & 1.343 & 0.018 & $195.8^{*} 0.8$ & & & & \\
\hline $20377+3322$ & STF2705 & 14078 & 2014.798 & $\mathrm{R}$ & 32 & 3.164 & 0.017 & $261.3^{*} 0.3$ & & & & \\
\hline $20378+6045$ & STF2717 & 14102 & 2014.831 & $\mathrm{RL}$ & 20 & 1.987 & 0.010 & $257.4^{*} 0.3$ & & & & \\
\hline $20396+4035$ & STT410 & 14126 & 2014.836 & $\mathrm{R}$ & 10 & 0.886 & 0.004 & $\begin{array}{ll}3.7 & 0.3\end{array}$ & & Hrt2011a & 0.02 & -0.1 \\
\hline $20410+3218$ & STF2716Aa-B & 14158 & 2014.836 & $\mathrm{RL}$ & 20 & 2.756 & 0.014 & $44.7^{*} 0.3$ & $\mathrm{NF}$ & & & \\
\hline $20423+5723$ & BU152 & 14196 & 2014.836 & $\mathrm{R}$ & 20 & 1.204 & 0.008 & $82.0^{*} 0.3$ & & & & \\
\hline $20450+1244$ & BU64 & 14238 & 2014.882 & $\mathrm{R}$ & 20 & 0.681 & 0.012 & $\begin{array}{ll}353.8 & 0.8\end{array}$ & $\mathrm{NF}$ & USN2007a & 0.03 & -1.4 \\
\hline $20474+3629$ & STT413AB & 14296 & 2014.825 & $\mathrm{R}$ & 20 & 0.910 & 0.008 & $2.6^{*} 0.3$ & & Rab1948b & -0.01 & 3.0 \\
\hline $20486+5029$ & BU366 & 14331 & 2014.882 & $\mathrm{R}$ & 32 & 1.399 & 0.025 & $128.5^{*} 0.8$ & & & & \\
\hline $20548+3242$ & STT418 & 14421 & 2014.893 & $\mathrm{R}$ & 32 & 0.932 & 0.017 & $282.8 \quad 0.3$ & & Zir2013a & -0.02 & -0.8 \\
\hline $20585+5028$ & STF2741AB & 14504 & 2014.798 & $\mathrm{R}$ & 32 & 1.954 & 0.017 & $25.2^{*} 0.3$ & & & & \\
\hline $21021+5640$ & STF2751 & 14575 & 2014.741 & $\mathrm{R}$ & 20 & 1.607 & 0.008 & $355.5^{*} 0.3$ & & & & \\
\hline $21022+0711$ & STF2742 & 14556 & 2014.893 & $\mathrm{R}$ & 32 & 2.960 & 0.020 & $213.6^{*} 0.3$ & Diffuse & & & \\
\hline $21118+5959$ & STF2780Aa-B & 14749 & 2014.741 & $\mathrm{R}$ & 20 & 1.027 & 0.008 & $212.9^{*} 0.3$ & & & & \\
\hline $21137+6424$ & H148 & 14783 & 2014.899 & $\mathrm{R}$ & 20 & 0.601 & 0.008 & $243.5^{*} 0.5$ & & Hrt2014b & -0.06 & 0.9 \\
\hline $21208+3227$ & STT437AB & 14889 & 2014.741 & $\mathrm{R}$ & 32 & 2.437 & 0.017 & $19.6^{*} 0.6$ & & Hrt2011a & 0.01 & 0.8 \\
\hline $21441+2845$ & STF2822AB & 15270 & 2014.836 & $\mathrm{R}$ & 20 & 1.649 & 0.008 & $317.3^{*} 0.3$ & & Hei1995 & 0.09 & -3.6 \\
\hline $21516+6545$ & STF2843AB & 15407 & 2014.893 & $\mathrm{R}$ & 20 & 1.326 & 0.014 & $149.7^{*} 0.3$ & & & & \\
\hline $21523+6306$ & STF2845AB & 15417 & 2014.883 & $\mathrm{R}$ & 32 & 1.893 & 0.020 & $173.0^{*} 0.6$ & & & & \\
\hline $21555+1053$ & BU75 & 15447 & 2014.836 & $\mathrm{R}$ & 32 & 1.016 & 0.017 & $23.8^{*} 0.9$ & & Hei1996a & -0.03 & -0.8 \\
\hline $21555+5232$ & STT456AB & 15460 & 2014.883 & $\mathrm{R}$ & 32 & 1.513 & 0.032 & $34.7^{*} 1.0$ & & & & \\
\hline$"$ & $"$ & $"$ & 2014.894 & $\mathrm{R}$ & 32 & 1.629 & 0.017 & $37.2^{*} 1.3$ & & & & \\
\hline $22044+1339$ & STF2854 & 15596 & 2014.798 & $\mathrm{R}$ & 32 & 1.579 & 0.017 & $81.6^{*} 0.4$ & & & & \\
\hline $22086+5917$ & STF 2872BC & 15670 & 2014.935 & $\mathrm{R}$ & 20 & 0.837 & 0.012 & 297.20 .4 & $\mathrm{NF}$ & USN2002 & 0.03 & 0.1 \\
\hline $22146+2934$ & STF2881 & 15769 & 2014.798 & $\mathrm{R}$ & 32 & 1.250 & 0.020 & $74.5^{*} 0.5$ & & & & \\
\hline $22288-0001$ & STF2909AB & 15971 & 2014.836 & $\mathrm{R}$ & 20 & 2.250 & 0.011 & $164.3^{*} 0.3$ & Diffuse & Sca2010c & 0.01 & -0.8 \\
\hline $22514+6142$ & STF2950 & 16317 & 2014.836 & $\mathrm{R}$ & 20 & 1.187 & 0.008 & $274.6^{*} 0.3$ & & Zir2015a & 0.00 & -0.2 \\
\hline $23595+3343$ & STF3050AB & 17149 & 2014.894 & $\mathrm{R}$ & 20 & 2.365 & 0.012 & $339.2^{*} 0.3$ & Diffuse & Hrt2011a & -0.00 & -0.2 \\
\hline
\end{tabular}

Note: In column $9, *$ indicates that $\theta$ was determined with our quadrant value (or with the long integration).

In column 14, the exponent ${ }^{Q}$ indicates discrepant quadrants between our measurements and the published orbits. 
Table 1). In that case, the bandpass and central wavelength correspond to that of the ICCD detector (see Prieur et al., 1998).

As for the other papers of this series, position measurements were obtained by an interactive processing of the autocorrelation files computed in real time during the observations. This processing led to a series of measurements with different background estimates and simulated noise, from which we derived the mean values and the standard deviation of those multiple measurements (see Paper III for more details). The final measures and their errors are displayed in Table 1. The average error values of the measurements reported in this table are $\left\langle\sigma_{\rho}\right\rangle=0^{\prime \prime} .015 \pm 0^{\prime \prime} .011$ and $\left\langle\sigma_{\theta}\right\rangle=0^{\circ} .5 \pm 1^{\circ} .4$.

\subsection{Quadrant determination}

As our astrometric measurements were obtained from the symmetric autocorrelation files, the $\theta$ values first presented a $180^{\circ}$ ambiguity. To resolve this ambiguity and determine the quadrant containing the companion, Aristidi et al. (1997) have proposed a method that can be considered as a restricted triple correlation (RTC hereafter). The quadrants of the measurements indicated in Table 1 were mostly derived from the RTC files that were computed in real time during the observations. However, for the couples with the largest separations, a straightforward determination was done when the companions could be directly spotted on the long integration files.

As a result, in Table 1, we are able to give the unambiguous (i.e. "absolute") position angles of 188 out of 224 measurements, i.e. $84 \%$ of the total. They are marked with an asterisk in Col 9. When our quadrant determination procedure failed, the angular measurement was reduced to the quadrant reported in the WDS catalog, which is extracted from the Fourth Catalog of Interferometric Measurements of Binary Stars (Hartkopf et al. 2016, hereafter IC4).

Our "absolute" $\theta$ values are consistent with the values tabulated in WDS for all objects except for ADS 4208, 10905 and 11558. We display some information about those objects in Table 2. In Col. 2, we indicate the quadrant $(\mathrm{Q})$ that we obtained from our observations, using the usual convention of numbering it from 1 to 4 to indicate the North-East, South-East, South-West and North-West quadrants, respectively. In Col. 3 we indicate which filter we have used. We report the difference of magnitude between the two components from the IC4 in Col. 4, and the global spectral type found in the SIMBAD astronomical data base in Col. 5. For those objects, the small value or the uncertainty in of the magnitude difference $\Delta m_{V}$ may account for the discrepancy.

In the case of triple systems, a new method to resolve the $180^{\circ}$ ambiguity is presented in Sect. 3.3 and 4 .
Table 2 Objects with discrepant quadrants

\begin{tabular}{lcclc}
\hline Name & Q & Filter & $\Delta m_{V}$ & Spectral type \\
\hline ADS 4208 & 2 & $\mathrm{R}$ & 0.01 & B9IV-V \\
ADS 10905 & 2 & $\mathrm{R}$ & - & A0III \\
ADS 11558 & 2 & $\mathrm{R}$ & 0.2 & A3V \\
\hline
\end{tabular}

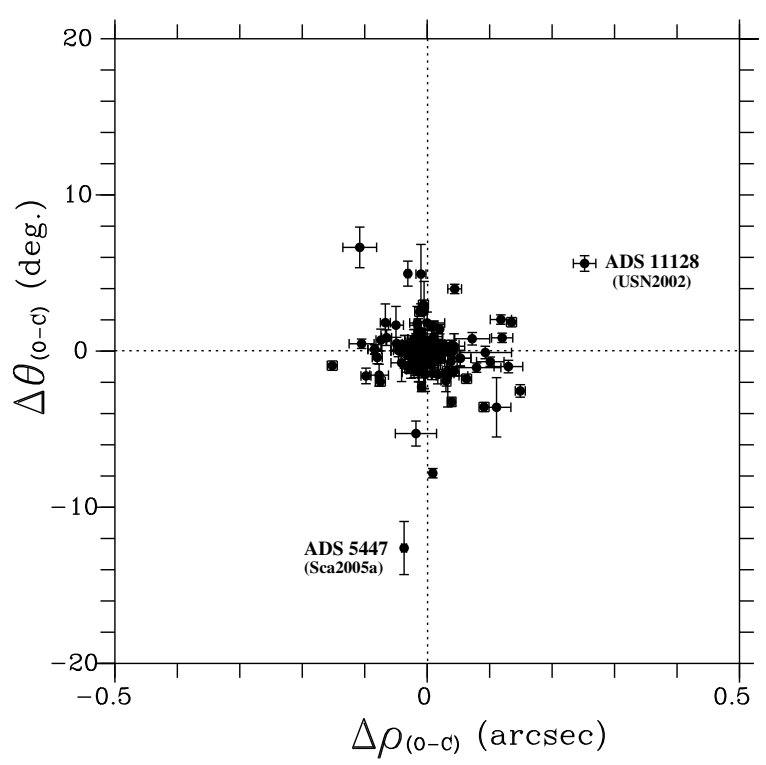

Fig. 3 Residuals of the measurements of Table 1 computed with the published orbits.

\subsection{Comparison with published ephemerides}

The $(O-C)$ (Observed minus Computed) residuals of the measurements for the systems with a known orbit in Table 1 are displayed in Cols. 13 and 14 for the separation $\rho$ and position angle $\theta$, respectively. Those residuals were obtained with a selection of valid orbits found in the OC6 catalog. We did not always use the most recent orbits since sometimes older orbits led to equivalent or even smaller residuals. For ADS 1615, 5447, 10075 and 12447, we also reported the residuals obtained with our revised orbit presented in Sect. 5 .

The residuals are plotted in Fig. 3. They have a rather large scatter which is naturally explained by the (old) age of many orbits that need revision. For example the large residuals obtained for ADS 5447 with our previous orbit (Scardia et al. 2005) have been largely reduced with our new revised orbit (see Sect. 5). The mean values computed with the residuals of Table 1 are $\left\langle\Delta \rho_{O-C}\right\rangle=0^{\prime \prime} .001 \pm 0^{\prime \prime} .05$ and $\left\langle\Delta \theta_{O-C}\right\rangle=-0^{\circ} .2 \pm 2^{\circ} .0$. The small values obtained for those offsets provide a good validation of our calibration (see Paper XII).

\subsection{Measurements of triple systems}

In Table 1, we have reported the measurements of the four triple systems that we observed in 2014: ADS 5400 

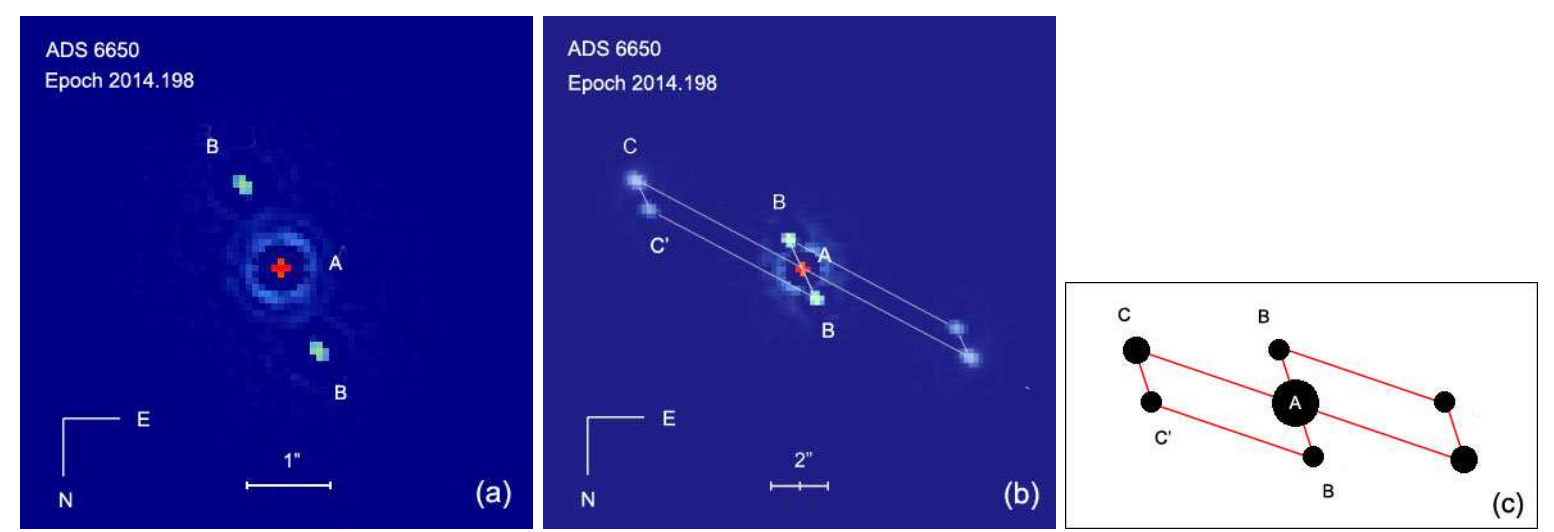

Fig. 4 Measurements of triple systems: case of ADS 6650. Autocorrelation obtained with a small field of view (a), and a wide field (b). As shown in (c), with the wide field autocorrelation, the AB (resp. AC) separation vector can also be determined with CC' (resp. BC').

(STF 948), ADS 6650 (STF 1196), ADS 6993 (STF 1273), and ADS 10227 (BU 627). In the literature, many measurements and orbits of triple systems refer to the couple AB-C. When the components AB are well separated the measurements (and the orbits) of $\mathrm{AB}-\mathrm{C}$ may be ambiguous, since the "center of AB" is defined by some authors as the center of light, or by others as the center of mass of the two components. To avoid this problem, we reported the $\mathrm{AC}$ measurements in Table 1, when the closest pair AB was resolved. For example, this is the case of ADS 6650, as shown in Fig. 4. This figure clearly shows that the separation and position angle of $\mathrm{CC}^{\prime}$ is the same as $\mathrm{AB}$. So CC' can be used to determine $A B$, if necessary.

Fig. 5 illustrates the different aspects of the autocorrelations of triple systems, obtained for the objects of the first two columns with a small field centered on the brightest component (Col. 3), or with a wide field including the 3 components (Col. 4). The objects in the first two columns are symmetric to one another, relative to the brightest component. As a result they have the same autocorrelation functions. This figure shows that the wide field autocorrelation (Col. 4) gives the relative position angle between close and wide pairs and thus contains useful information about the absolute position angle of the closest pair. An example of application will be given in the next section.

\section{Study of the multiple system ADS 6993 with PISCO in Merate (2004-2014)}

\subsection{Description of the system}

SP $1(\epsilon$ Hya $=11$ Hya $)$ : the AB pair was discovered at the Osservatorio Astronomico di Brera in 1888 by Schiaparelli with the $50 \mathrm{~cm}$ Merz-Repsold refractor (Schiaparelli, 1909). In fact, this object is a multiple system from which two couples could be observed in
Merate: SP1AB and STF1273AB,C. The other components of this sytem are beyond the capabilities of our instrument, and could not be observed.

\subsection{Observations with PISCO since 2004 and first data reduction}

SP $1 \mathrm{AB}$ was observed in Merate in 2004, 2007, 2010, 2012, and 2014, with the $10 \mathrm{~mm}$ eyepiece (see Table 3). The observations of 2004 appeared first as unresolved (Paper I), because its separation was close to the diffraction limit of the Zeiss telescope. A subsequent processing of the same observations, by subtracting a model obtained from a single (or unresolved) star, allowed us to resolve this pair (see Paper IV). The AB pair was then directly resolved in our subsequent smallfield observations with the $10 \mathrm{~mm}$ eyepiece.

STF1273AB,C was observed with the $20 \mathrm{~mm}$ eyepiece in 2007,2010,2012, and 2014, at the same epochs as SP $1 \mathrm{AB}$ (see Table 3). In papers VII, XI, and XIII, we reported $\mathrm{AB}, \mathrm{C}$ measurements since the $\mathrm{AB}$ pair appeared (in that time) as unresolved in those lowmagnification observations.

\subsection{New data reduction}

In 2014, we improved our data reduction pipeline and implemented new high-contrast numerical filters based on strioscopy (elimination of the low frequency components of the image). When re-processing the wide-field autocorrelations of ADS 6993 with those filters, the two secondary peaks appeared as resolved into two tiny spots of different brightness. Furthermore, by subtracting a model of the background in the central regions, it was possible to detect the $\mathrm{A}$ and $\mathrm{B}$ components, for the observations of 2010, 2012 and 2014 (Fig. 6). In those cases, the secondary peaks became sixfold!

Since SP $1 \mathrm{AB}$ was resolved in the small-field autocorrelations obtained with the $10 \mathrm{~mm}$ eyepiece, it appears that ADS 6993 is similar to the objects 4 or 

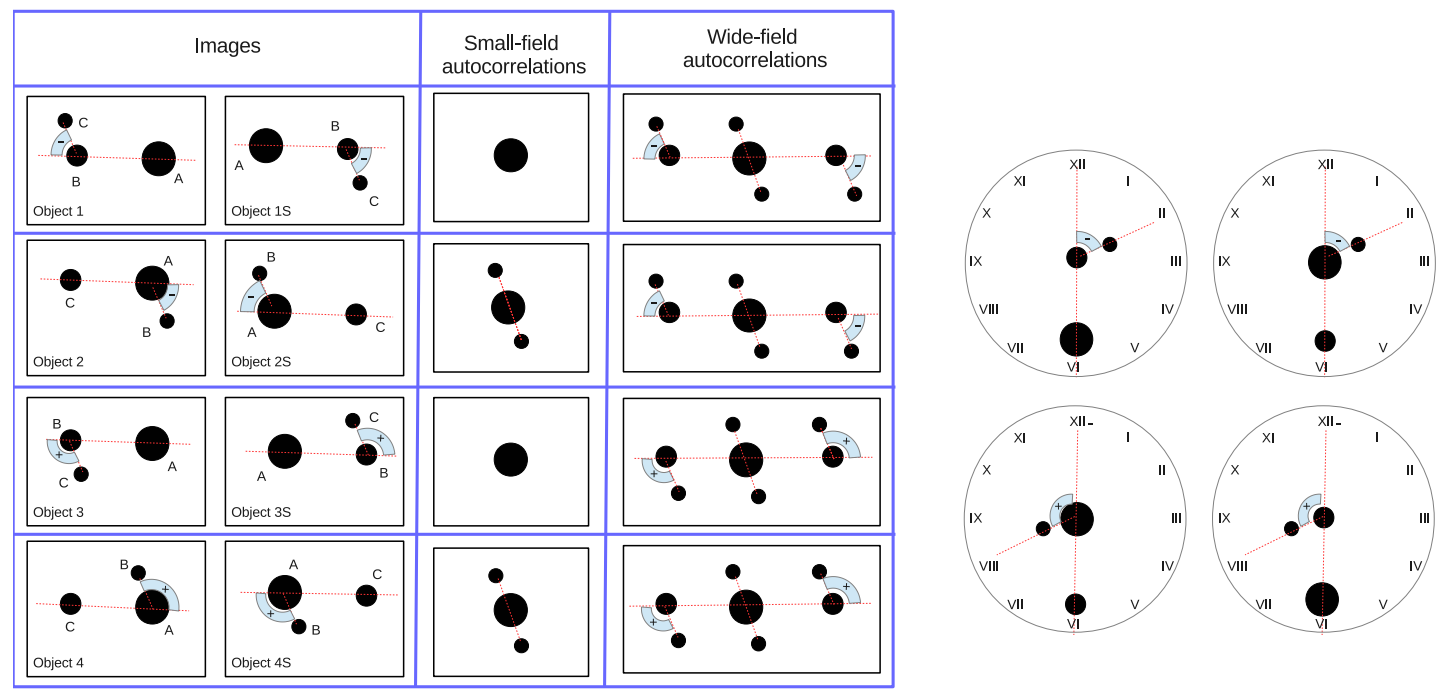

Fig. 5 Triple systems : the $180^{\circ}$ ambiguity of the position angle of the companion of the closest pair can be resolved by examining the secondary peaks of the wide-field autocorrelation function. This is illustrated with the virtual clocks on the right, where the isolated component in the image plane (or the central peak of the autocorrelation) is drawn at 6 p.m.
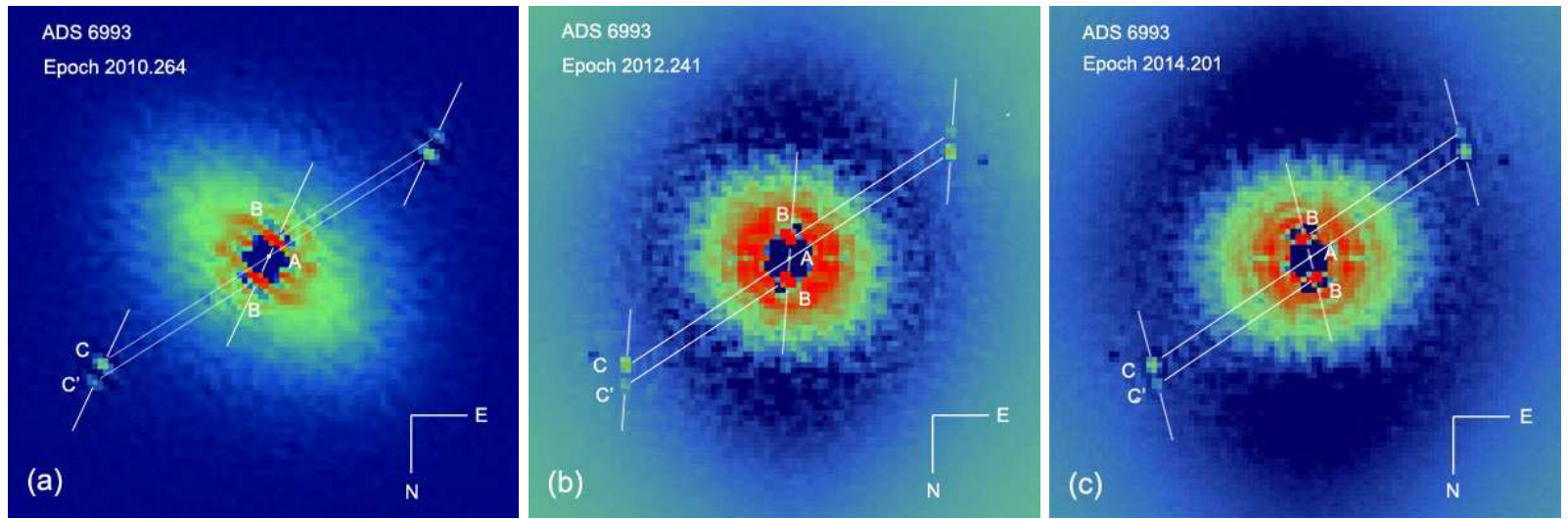

Fig. 6 ADS 6993: in the wide-field autocorrelations obtained in 2010 (a), 2012 (b) and 2014 (c), the couple AB appears both as secondary peaks close to the center, and as a splitting of the distant secondary peaks produced by the C component.

$4 \mathrm{~S}$ of Fig. 5. As the position angle of the $\mathrm{AC}$ pair is $\theta=304^{\circ} .5$ (see Table 1 ), we infer that we are here in the case of object 4 . Therefore, the image of the pair $\mathrm{AB}$ looks like the upper right part of the autocorrelations of Fig. 6: from 2010 to 2014, the B component has moved from the second to the third quadrant. This in agreement with other published measurements (see IC4), and with an image of the ABC components obtained in Lucky-imaging by R. Gili in 2012 (private communication).

Our results are displayed in Table 3 . The measurements of SP $1 \mathrm{AB}$ obtained with the $10 \mathrm{~mm}$ eyepiece (small-field autocorrelations) are in very good agreement with those obtained when measuring the separation $\mathrm{CC}^{\prime}$ in the wide-field autocorrelations. Due to the small orbital period ( $P \approx 15 \mathrm{yr}$ ), the auto-correlation images have clearly changed between 2010 and 2014 (see Fig. 6).

\section{Revised orbits of ADS 671, 1615, $1709,5447,10075$ and 12447}

In this section we present the new revised orbits that we have computed for ADS 671, 1615, 1709, 5447, 10075 and 12447 . The revision of those orbits was justified by the appearance of a systematic trend in the residuals of our last measurements and/or the existence of substantial number of new measurements since the computation of the last known orbit. The orbital elements of the revised orbits were published in the circulars of the IAU Commission 26 (Scardia, 2015b, 2015c) and are in the Sixth Catalogue of Orbits of Visual Binary Stars (Hartkopf \& Mason, 2016) under reference Scar2015c.

We have followed the same method for computing the orbits of those seven objects. Using our last measurements with PISCO and the other available observations contained in the data base maintained by the United States Naval Observatory (USNO), we first 
Table 3 Observations of ADS 6993 with PISCO since 2004: old and new measurements.

\begin{tabular}{|c|c|c|c|c|c|c|c|c|c|c|c|}
\hline Name & Epoch & Fil. & $\begin{array}{l}\text { Eyep. } \\
(\mathrm{mm})\end{array}$ & $\begin{array}{l}\rho \\
\left({ }^{\prime \prime}\right)\end{array}$ & $\begin{array}{l}\sigma_{\rho} \\
\left({ }^{\prime \prime}\right)\end{array}$ & $\begin{array}{r}\theta \\
\left({ }^{\circ}\right)\end{array}$ & $\begin{array}{l}\sigma_{\theta} \\
\left({ }^{\circ}\right)\end{array}$ & Orbit & $\Delta \rho(\mathrm{O}-\mathrm{C})$ & $\Delta \theta(\mathrm{O}-\mathrm{C})$ & Notes \\
\hline SP $1 \mathrm{AB}$ & 2004.208 & $\mathrm{~V}$ & 10 & 0.195 & 0.004 & 258.5 & 2.7 & Hrt1999a & -0.02 & +1.4 & New measure (data from Paper IV) \\
\hline $\mathrm{SP} 1 \mathrm{AB}$ & 2007.198 & $\mathrm{~V}$ & 10 & 0.151 & 0.004 & 108.1 & 0.6 & Hrt1999a & +0.00 & +1.3 & Paper VII \\
\hline $\mathrm{SP} 1 \mathrm{AB}$ & 2007.198 & $\mathrm{R}$ & 20 & - & - & - & - & & & & Paper VII : unresolved \\
\hline STF1273CC' & 2007.198 & $\mathrm{R}$ & 20 & - & - & - & - & & & & Unresolved (data from Paper VII) \\
\hline STF1273AB,C & 2007.198 & $\mathrm{R}$ & 20 & 2.767 & 0.014 & 301.5 & 0.3 & Dru2014 & -0.05 & +0.3 & See note ${ }^{(1)}$ \\
\hline $\mathrm{SP} 1 \mathrm{AB}$ & 2010.264 & $\mathrm{R}$ & 10 & 0.259 & 0.004 & 154.8 & 0.6 & Hrt1999a & -0.01 & -0.9 & New measure (data from Paper XI) \\
\hline STF1273CC' & 2010.264 & $\mathrm{R}$ & 20 & 0.289 & 0.004 & 154.3 & 0.4 & $"$ & +0.03 & -1.4 & New measure (data from Paper XI) \\
\hline STF1273AB,C & 2010.264 & RL & 20 & 2.765 & 0.018 & 302.9 & 0.5 & Dru2014 & -0.06 & -0.6 & New measure (data from Paper XI) \\
\hline STF1273AC & 2010.264 & $\mathrm{R}$ & 20 & 2.727 & 0.008 & 302.4 & 0.3 & & & & New measure (data from Paper XI) \\
\hline SP $1 \mathrm{AB}$ & 2012.241 & $\mathrm{R}$ & 10 & 0.269 & 0.003 & 176.2 & 1.6 & Hrt1999a & -0.01 & +0.2 & Paper XIII \\
\hline $\mathrm{SP} 1 \mathrm{AB}$ & 2012.241 & $\mathrm{R}$ & 20 & 0.307 & 0.008 & 177.1 & 0.9 & $"$ & +0.03 & +1.1 & New measure (data from Paper XIII) \\
\hline STF1273CC' (2) & 2012.241 & $\mathrm{R}$ & 20 & 0.301 & 0.009 & 178.1 & 0.4 & $"$ & +0.03 & +2.1 & New measure (data from Paper XIII) \\
\hline STF1273AB,C & 2012.241 & $\mathrm{RL}$ & 20 & 2.756 & 0.017 & 303.4 & 0.3 & Dru2014 & -0.06 & -1.5 & See note ${ }^{(3)}$ \\
\hline STF1273AC & 2012.241 & $\mathrm{R}$ & 20 & 2.741 & 0.008 & 303.1 & 0.3 & & & & New measure (data from Paper XIII) \\
\hline SP $1 \mathrm{AB}$ & 2014.201 & $\mathrm{R}$ & 10 & 0.261 & 0.003 & 194.0 & 0.5 & Hrt1999a & -0.01 & -1.8 & New data \\
\hline $\mathrm{SP} 1 \mathrm{AB}$ & 2014.201 & $\mathrm{R}$ & 20 & 0.294 & 0.008 & 195.7 & 0.6 & , & +0.02 & -0.1 & $"$ \\
\hline STF1273CC' & 2014.201 & $\mathrm{R}$ & 20 & 0.287 & 0.008 & 193.8 & 0.4 & $"$ & +0.02 & -2.0 & $"$ \\
\hline STF1273AB,C & 2014.201 & $\mathrm{RL}$ & 20 & 2.786 & 0.009 & 305.2 & 0.3 & Dru2014 & -0.03 & -1.2 & $"$ \\
\hline STF1273AC & 2014.201 & $\mathrm{R}$ & 20 & 2.768 & 0.008 & 304.5 & 0.3 & & & & $"$ \\
\hline
\end{tabular}

(1) Reported in IC4 as 1273AC (Pru 2009) although it was published in Paper VII as 1273AB-C.

(2) STF1273CC' is an independant (unambiguous) measurement of SP1AB (see Fig 4)

(3) Published in Paper XIII as STF1273AC.

Table 4 New orbital elements of ADS 671, 1615, 1709, 5447, 10075 and 12447.

\begin{tabular}{|c|c|c|c|c|c|c|c|c|c|c|c|c|}
\hline ADS & $\begin{array}{c}\Omega_{2000} \\
\left({ }^{\circ}\right)\end{array}$ & $\begin{array}{c}\omega \\
\left(^{\circ}\right)\end{array}$ & $\begin{array}{c}i \\
\left(^{\circ}\right)\end{array}$ & $e$ & $\begin{array}{c}T \\
(\mathrm{yr})\end{array}$ & $\begin{array}{c}P \\
(\mathrm{yr})\end{array}$ & $\begin{array}{c}n \\
\left({ }^{\circ} / \mathrm{yr}\right)\end{array}$ & $\begin{array}{c}a \\
\left({ }^{\prime \prime}\right)\end{array}$ & $\begin{array}{c}\mathrm{A} \\
\left({ }^{\prime \prime}\right)\end{array}$ & $\begin{array}{c}\mathrm{B} \\
\left({ }^{\prime \prime}\right)\end{array}$ & $\begin{array}{c}\mathrm{F} \\
\left({ }^{\prime \prime}\right)\end{array}$ & $\begin{array}{c}\mathrm{G} \\
\left({ }^{\prime \prime}\right)\end{array}$ \\
\hline 671 & $\begin{array}{c}278.0 \\
\pm 0.6\end{array}$ & $\begin{array}{c}269.8 \\
\pm 1.0\end{array}$ & $\begin{array}{l}35.6 \\
\pm 0.3\end{array}$ & $\begin{array}{c}0.497 \\
\pm 0.006\end{array}$ & $\begin{array}{c}1889.93 \\
\pm 0.18\end{array}$ & $\begin{array}{c}479.27 \\
\pm 8.2\end{array}$ & $\begin{array}{l}0.75114 \\
\pm 0.0013\end{array}$ & $\begin{array}{l}12.04 \\
\pm 0.02\end{array}$ & -9.70273 & -1.32079 & 1.64217 & 11.92746 \\
\hline 1615 & $\begin{array}{l}3.70 \\
-\end{array}$ & $\begin{array}{c}147.9 \\
-\end{array}$ & $\begin{array}{c}113.4 \\
-\end{array}$ & $\begin{array}{c}0.465 \\
-\end{array}$ & $\begin{array}{c}2188.6 \\
-\end{array}$ & $\begin{array}{c}3267.4 \\
-\end{array}$ & $\begin{array}{c}0.1102 \\
-\end{array}$ & $\begin{array}{l}7.4 \\
-\end{array}$ & -6.15485 & -1.96300 & -4.08482 & 2.23065 \\
\hline 1709 & $\begin{array}{l}99.07 \\
\pm 0.25\end{array}$ & $\begin{array}{c}322.66 \\
\pm 0.60\end{array}$ & $\begin{array}{l}63.75 \\
\pm 0.17\end{array}$ & $\begin{array}{c}0.2626 \\
\pm 0.0016\end{array}$ & $\begin{array}{c}1899.07 \\
\pm 0.21\end{array}$ & $\begin{array}{l}145.41 \\
\pm 0.47\end{array}$ & $\begin{array}{c}2.4758 \\
\pm 0.0080\end{array}$ & $\begin{array}{c}0.89 \\
\pm 0.02\end{array}$ & 0.12353 & 0.73225 & -0.39193 & 0.48102 \\
\hline 5447 & $\begin{array}{l}10.1 \\
\pm 12\end{array}$ & $\begin{array}{c}258.9 \\
\pm 8.5\end{array}$ & $\begin{array}{l}150.5 \\
\pm 4.8\end{array}$ & $\begin{array}{c}0.605 \\
\pm 0.031\end{array}$ & $\begin{array}{c}2017.76 \\
\pm 0.51\end{array}$ & $\begin{array}{c}205.3 \\
\pm 8.2\end{array}$ & $\begin{array}{c}1.75315 \\
\pm 0.070\end{array}$ & $\begin{array}{c}0.45 \\
\pm 0.02\end{array}$ & -0.15100 & 0.35915 & 0.41683 & 0.14999 \\
\hline 10075 & $\begin{array}{c}93.86 \\
\pm 0.10\end{array}$ & $\begin{array}{l}130.75 \\
\pm 0.19\end{array}$ & $\begin{array}{l}108.42 \\
\pm 0.11\end{array}$ & $\begin{array}{c}0.7584 \\
\pm 0.0022\end{array}$ & $\begin{array}{c}1921.116 \\
\pm 0.021\end{array}$ & $\begin{array}{c}230.06 \\
\pm 3.0\end{array}$ & $\begin{array}{l}1.56481 \\
\pm 0.020\end{array}$ & $\begin{array}{c}2.25 \\
\pm 0.05\end{array}$ & 0.63483 & -1.42594 & -0.34751 & -1.72805 \\
\hline 12447 & $\begin{array}{c}155.3 \\
-\end{array}$ & $\begin{array}{c}62.5 \\
-\end{array}$ & $\begin{array}{c}150.7 \\
-\end{array}$ & $\begin{array}{c}0.958 \\
-\end{array}$ & $\begin{array}{c}1887.722 \\
-\end{array}$ & $\begin{array}{c}882.894 \\
-\end{array}$ & $\begin{array}{c}0.4078 \\
-\end{array}$ & $\begin{array}{c}1.87 \\
-\end{array}$ & -0.18002 & 1.67498 & 1.82161 & -0.00901 \\
\hline
\end{tabular}

computed the preliminary orbital elements with the analytical method of Kowalsky (1873). We then used them as initial values for the least-squares method of Hellerich (1925). When convergence was achieved, Hellerich's method led to an improvement of the orbital elements (with the exception of the major axis) and to an estimation of the corresponding errors. The final value of the major axis was then set to the value that minimized the residuals in separation of Hellerich's solution.

The final orbital elements are presented in Table 4. The errors reported in this table were obtained by
Hellerich's least-squares method. For ADS 1615 and 12447, the errors could not be estimated since Hellerich's method did not converge. The format of the tables contained in this section is self-explanatory, but a detailed description of those formats can be found in Papers VI and VII.

The $(O-C)$ residuals of the new orbits, restricted to the last observations for reasons of space, are given in Tables $5,6,7,8,9,10$. The name of the observer is reported in the last column, using the US Naval Observatory convention. 
Table 5 ADS 671: O-C residuals of our new orbit (after 2011).

\begin{tabular}{crrl}
\hline Epoch & $\begin{array}{r}\Delta \rho(\mathrm{O}-\mathrm{C}) \\
\left({ }^{\prime \prime}\right)\end{array}$ & $\Delta \theta(\mathrm{O}-\mathrm{C})$ & Observer \\
& 0.009 & -0.178 & $\mathrm{Mwl}$ \\
\hline 2012.766 & 0.018 & 0.107 & $\mathrm{Smr}$ \\
2012.801 & 0.025 & 0.112 & $\mathrm{Mwl}$ \\
2013.922 & 0.045 & 0.111 & $\mathrm{Mwl}$ \\
2013.924 & 0.334 & 0.095 & Ary \\
2014.641 & & \\
\hline
\end{tabular}

Table 6 ADS 1615: O-C residuals of our new orbit (after 2012). The symbol ${ }^{P}$ indicates PISCO measurements.

\begin{tabular}{crrl}
\hline Epoch & $\begin{array}{r}\Delta \rho(\mathrm{O}-\mathrm{C}) \\
\left({ }^{\prime \prime}\right)\end{array}$ & $\begin{array}{r}\Delta \theta(\mathrm{O}-\mathrm{C}) \\
\left({ }^{\circ}\right)\end{array}$ & Observer \\
\hline 2012.015 & -0.151 & 0.687 & WSI \\
2012.038 & $-0.001^{P}$ & $0.201^{P}$ & Pru \\
2014.042 & $-0.019^{P}$ & $0.164^{P}$ & Sca \\
2015.017 & $-0.008^{P}$ & $-0.122^{P}$ & Sca \\
2015.112 & $0.006^{P}$ & $-0.163^{P}$ & Sca \\
\hline
\end{tabular}

The ephemerides for 2017-2025 are presented in Table 11. The apparent orbits are shown in Fig. 7 as solid lines. The observational data used for the calculation of the orbital elements are plotted as small crosses or, in the case of PISCO observations, as filled circles (that appear in red in the electronic version). The orientation of the graphs conforms to the convention adopted by the observers of visual binary stars. For each object, the location of the primary component is indicated with a big cross. The straight line going through this point is the line of apsides. An arrow shows the sense of rotation of the companion.

In Table 12, we present some physical parameters of those systems. The (total) visual magnitudes (Col. 3) and the spectral types (Col. 5) were extracted from the SIMBAD data base. The difference of magnitude between the components (Col. 4) was taken from the IC4. The dynamical parallaxes (Col. 6) were derived from our orbital elements using Baize \& Romani (1946)'s method, with our revised formulae presented in Scardia et al. (2008b). In Col. 7, we report the Hipparcos parallaxes from ESA (1997) or the revised values from van Leeuwen (2007), as indicated in Col. 11. In Cols. 8, 9 and 10, we give the corresponding angular and linear sizes of the semi-major axis $a$ and the total mass $\mathfrak{M}_{\text {total }}$, respectively, that were computed from our orbital elements and the Hipparcos parallaxes.

\section{WDS 00491+5749 - STF 60AB - ADS 671 - HIP 3821 ( $\eta$ Cas)}

This couple was discovered by W. Herschel on Aug. 18th 1779 (Herschel, 1782) with his famous Newtonian telescope $(\mathrm{D}=0.16 \mathrm{~m}, \mathrm{~F}=2.1 \mathrm{~m})$ that he built himself and installed in the backyard of his house of Bath. While commenting his numerous observations in the
Table 7 ADS 1709: O-C residuals of our new orbit (after 2009.7). The symbol ${ }^{P}$ indicates PISCO measurements.

\begin{tabular}{ccrl}
\hline Epoch & $\begin{array}{r}\Delta \rho(\mathrm{O}-\mathrm{C}) \\
\left({ }^{\prime \prime}\right)\end{array}$ & $\begin{array}{r}\Delta \theta(\mathrm{O}-\mathrm{C}) \\
\left(^{\circ}\right)\end{array}$ & Observer \\
\hline 2009.829 & -0.030 & 1.436 & Los \\
2009.908 & -0.033 & 1.735 & FMR \\
2010.777 & -0.044 & 3.505 & Ant \\
2012.043 & $-0.026^{P}$ & $-0.400^{P}$ & Pru \\
2015.113 & $-0.023^{P}$ & $-0.053^{P}$ & Sca \\
\hline
\end{tabular}

Table 8 ADS 5447: O-C residuals of our new orbit (after 2004). The symbol ${ }^{P}$ indicates PISCO measurements.

\begin{tabular}{cccl}
\hline Epoch & $\begin{array}{r}\Delta \rho(\mathrm{O}-\mathrm{C}) \\
\left({ }^{\prime \prime}\right)\end{array}$ & $\begin{array}{r}\Delta \theta(\mathrm{O}-\mathrm{C}) \\
\left(^{\circ}\right)\end{array}$ & Observer \\
\hline 2004.211 & $0.001^{P}$ & $1.038^{P}$ & Sca \\
2005.136 & $-0.002^{P}$ & $0.820^{P}$ & Sca \\
2007.200 & $0.003^{P}$ & $1.144^{P}$ & Pru \\
2008.240 & $0.011^{P}$ & $2.671^{P}$ & Pru \\
2013.890 & $0.017^{P}$ & 3.869 & OCC \\
2014.239 & $0.012^{P}$ & $-1.900^{P}$ & Pru \\
\hline
\end{tabular}

Table 9 ADS 10075: O-C residuals of our new orbit (after 2012). The symbol ${ }^{P}$ indicates PISCO measurements.

\begin{tabular}{ccrl}
\hline Epoch & $\begin{array}{r}\Delta \rho(\mathrm{O}-\mathrm{C}) \\
\left({ }^{\prime \prime}\right)\end{array}$ & $\begin{array}{r}\Delta \theta(\mathrm{O}-\mathrm{C}) \\
\left(^{\circ}\right)\end{array}$ & Observer \\
\hline 2012.460 & $-0.029^{P}$ & $-0.628^{P}$ & Pru \\
2012.516 & -0.072 & 0.389 & Wly \\
2013.296 & 0.022 & -0.372 & RAO \\
2013.583 & -0.015 & 2.116 & Ary \\
2013.601 & 0.012 & -0.679 & RAO \\
2014.511 & $-0.005^{P}$ & $-0.605^{P}$ & Sca \\
\hline
\end{tabular}

Table 10 ADS 12447: O-C residuals of our new orbit (after 2011). The symbol ${ }^{P}$ indicates PISCO measurements.

\begin{tabular}{cccl}
\hline Epoch & $\begin{array}{r}\Delta \rho(\mathrm{O}-\mathrm{C}) \\
\left({ }^{\prime \prime}\right)\end{array}$ & $\begin{array}{r}\Delta \theta(\mathrm{O}-\mathrm{C}) \\
\left({ }^{\circ}\right)\end{array}$ & Observer \\
\hline 2011.663 & $0.027^{P}$ & $-0.530^{P}$ & Sca \\
2011.823 & - & 1.893 & Pal \\
2011.842 & -0.215 & -0.565 & Pal \\
2013.630 & -0.000 & -0.767 & RAO \\
2013.733 & $0.076^{P}$ & $-1.058^{P}$ & Sca \\
2014.740 & $0.020^{P}$ & $-0.274^{P}$ & Sca \\
\hline
\end{tabular}

Philosophical Transactions (Herschel, 1804), he noticed a significant relative motion: "...which gives a change of $8^{\circ} 42$ ', in 20 years and 242 days. This arises probably from a real motion of eta in space...". More historical information about this couple can be found in the "Handbook of double stars" (Crossley et al. 1879).

This very wide couple is easily resolved by small telescopes and was extensively observed. More than 1100 observations are reported in the WDS data base. 

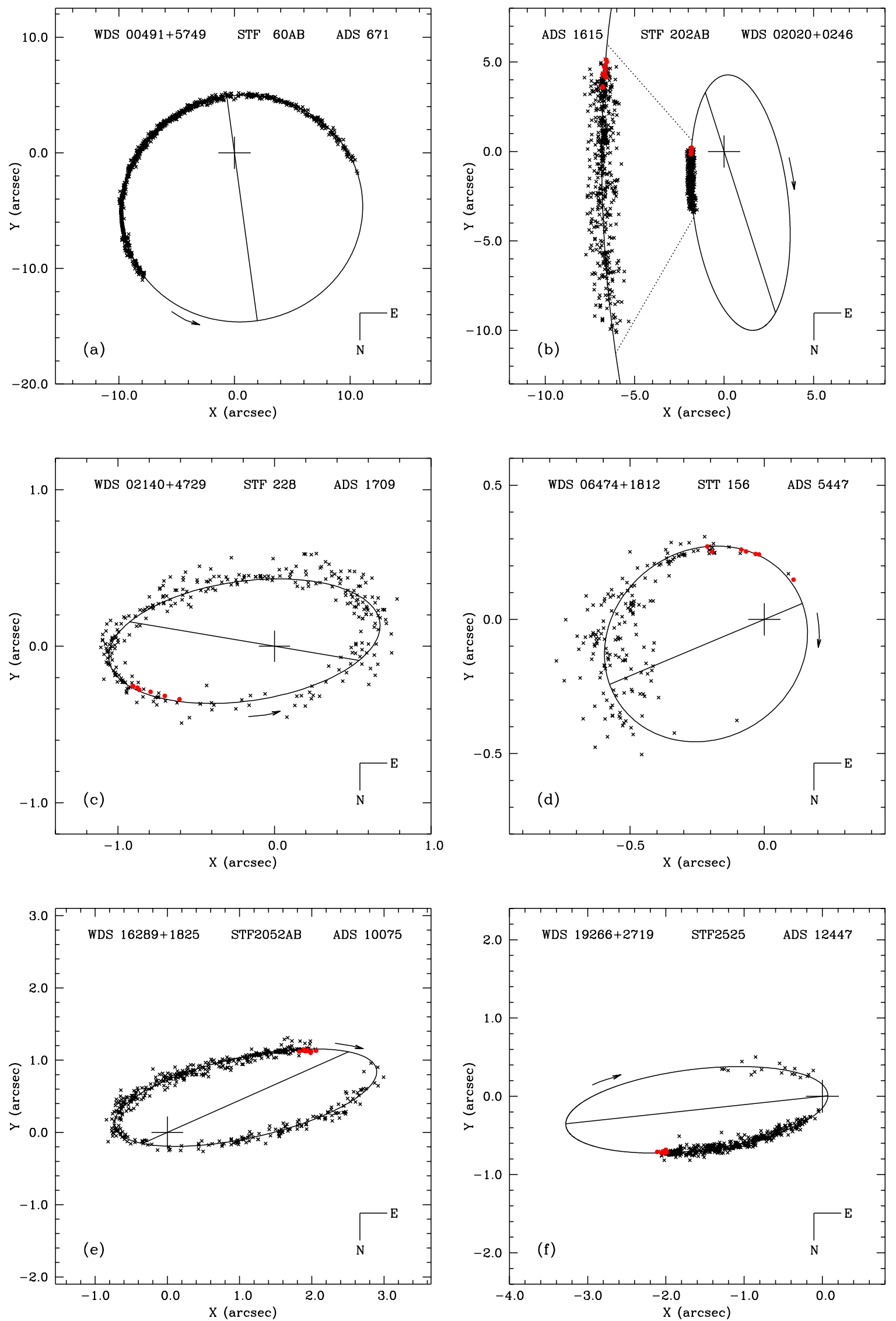

Fig. 7 New orbits of ADS 671 (a), ADS 1615 (b), ADS 1709 (c), ADS 5447 (d), ADS 10075 (e) and ADS 12447 (f). The observations by PISCO are plotted as filled circles that appear in red in the electronic version of this paper. 
Table 11 New ephemerides of ADS 671, 1615, 1709, 5447, 10075 and 12447.

\begin{tabular}{|c|c|c|c|c|c|c|c|c|c|c|c|c|}
\hline \multirow[b]{2}{*}{ Epoch } & \multicolumn{2}{|c|}{ ADS 671} & \multicolumn{2}{|c|}{ ADS 1615} & \multicolumn{2}{|c|}{ ADS 1709} & \multicolumn{2}{|c|}{ ADS 5447} & \multicolumn{2}{|c|}{ ADS 10075} & \multicolumn{2}{|c|}{ ADS 12447} \\
\hline & $\begin{array}{c}\rho \\
\left({ }^{\prime \prime}\right)\end{array}$ & $\begin{array}{c}\theta \\
\left(^{\circ}\right)\end{array}$ & $\begin{array}{c}\rho \\
\left({ }^{\prime \prime}\right)\end{array}$ & $\begin{array}{c}\theta \\
\left(^{\circ}\right)\end{array}$ & $\begin{array}{c}\rho \\
\left({ }^{\prime \prime}\right)\end{array}$ & $\begin{array}{c}\theta \\
\left(^{\circ}\right)\end{array}$ & $\begin{array}{c}\rho \\
\left({ }^{\prime \prime}\right)\end{array}$ & $\begin{array}{c}\theta \\
\left(^{\circ}\right)\end{array}$ & $\begin{array}{c}\rho \\
\left({ }^{\prime \prime}\right)\end{array}$ & $\begin{array}{c}\theta \\
\left(^{\circ}\right)\end{array}$ & $\begin{array}{c}\rho \\
\left({ }^{\prime \prime}\right)\end{array}$ & $\begin{array}{c}\theta \\
\left(^{\circ}\right)\end{array}$ \\
\hline 2017 & 3.323 & 25.0 & 834 & 62.0 & 667 & 302.4 & 156 & 120.4 & .407 & 118.7 & 2.181 & 289.4 \\
\hline 2018.0 & 13.351 & 325.4 & 1.835 & 261.4 & 0.639 & 304.4 & 0.154 & 110.3 & 2.427 & 118.4 & 2.189 & 289.3 \\
\hline 2019.0 & 13.378 & 325.9 & 1.836 & 260.8 & 0.611 & 306.5 & 0.154 & 100.1 & 2.448 & 118.1 & 2.198 & 289.2 \\
\hline 2020.0 & 13.405 & 326.3 & 1.838 & 260.1 & 0.582 & 308.8 & 0.157 & 90.1 & 2.467 & 117.9 & 2.207 & 289.2 \\
\hline 2021.0 & 13.431 & 326.7 & 1.839 & 259.5 & 0.553 & 311.4 & 0.162 & 80.7 & 2.487 & 117.6 & 2.215 & 289.1 \\
\hline 2022.0 & 13.458 & 327.1 & 1.841 & 258.9 & 0.523 & 314.3 & 0.170 & 71.9 & 2.506 & 117.3 & 2.224 & 289.0 \\
\hline 2023.0 & 13.483 & 327.6 & 1.843 & 258.3 & 0.494 & 317.5 & 0.178 & 64.0 & 2.525 & 117.1 & 2.232 & 289.0 \\
\hline 2024.0 & 13.509 & 328.0 & 1.846 & 257.6 & 0.465 & 321.1 & 0.188 & 56.8 & 2.544 & 116.8 & 2.240 & 288.9 \\
\hline 2025.0 & 13.534 & 328.4 & 1.848 & 257.0 & 0.437 & 325.1 & 0.199 & 50.4 & 2.562 & 116.6 & 2.249 & 288.8 \\
\hline
\end{tabular}

Table 12 Physical parameters $\left(\pi_{\text {dyn }}, a\right.$ and $\left.\mathfrak{M}_{\text {total }}\right)$ derived from the new orbital elements.

\begin{tabular}{|c|c|c|c|c|c|c|c|c|c|c|}
\hline ADS & HIP & $m_{V}$ & $\Delta m_{V}$ & Spectral type & $\begin{array}{c}\pi_{\mathrm{dyn}} \\
(\mathrm{mas})\end{array}$ & $\begin{array}{c}\pi_{\mathrm{HIP}} \\
(\mathrm{mas})\end{array}$ & $\left({ }^{\prime \prime}\right)$ & $\begin{array}{l}a \\
(\mathrm{AU})\end{array}$ & $\begin{array}{r}\mathfrak{M}_{\text {total }} \\
\left(\mathrm{M}_{\odot}\right)\end{array}$ & Source of $\pi_{\text {HIP }}$ \\
\hline 671 & 3821 & 3.52 & 3.8 & $\mathrm{G} 0 \mathrm{~V}+\mathrm{K} 7 \mathrm{~V}$ & 170.4 & $\begin{array}{c}167.99 \\
\pm 0.62\end{array}$ & 12.04 & $\begin{array}{l}71.7 \\
\pm 0.3\end{array}$ & $\begin{array}{r}1.60 \\
\pm 0.06\end{array}$ & ESA (1997) \\
\hline$"$ & $"$ & $"$ & $"$ & $"$ & $"$ & $\begin{array}{c}167.99 \\
\pm 0.62\end{array}$ & $"$ & $\begin{array}{l}71.7 \\
\pm 0.3\end{array}$ & $\begin{array}{r}1.60 \\
\pm 0.06\end{array}$ & van Leeuwen (2007) \\
\hline 1615 & 9487 & 3.82 & 1.1 & F2 IV & 20.0 & $\begin{array}{l}23.45 \\
\pm 1.06\end{array}$ & 7.4 & $\begin{array}{l}315 \\
\pm 14^{(1)}\end{array}$ & $\begin{array}{c}2.9 \\
\pm 0.4^{(1)}\end{array}$ & ESA (1997) \\
\hline$"$ & $"$ & $"$ & $"$ & $"$ & $"$ & $\begin{array}{l}21.66 \\
\pm 1.06\end{array}$ & $"$ & $\begin{array}{l}342 \\
\pm 17^{(1)}\end{array}$ & $\begin{array}{c}3.7 \\
\pm 0.6^{(1)}\end{array}$ & van Leeuwen (2007) \\
\hline 1709 & 10403 & 6.08 & 0.6 & $\mathrm{~F} 4 \mathrm{~V}$ & 23.6 & $\begin{array}{l}24.07 \\
\pm 0.96\end{array}$ & 0.89 & $\begin{array}{r}36.8 \\
\pm 1.7\end{array}$ & $\begin{array}{r}2.4 \\
\pm 0.3\end{array}$ & ESA (1997) \\
\hline$"$ & $"$ & $"$ & $"$ & $"$ & $"$ & $\begin{array}{l}25.26 \\
\pm 0.66\end{array}$ & $"$ & $\begin{array}{r}35.0 \\
\pm 1.3\end{array}$ & $\begin{array}{r}2.0 \\
\pm 0.2\end{array}$ & van Leeuwen (2007) \\
\hline 5447 & 32539 & 6.20 & 1.4 & $\mathrm{~A} 2 \mathrm{~V}$ & 8.0 & $\begin{array}{l}7.56 \\
\pm 1.41\end{array}$ & 0.45 & $\begin{array}{r}59 \\
\pm 11\end{array}$ & $\begin{array}{r}4.8 \\
\pm 2.8\end{array}$ & ESA (1997) \\
\hline$"$ & $"$ & $"$ & $"$ & $"$ & $"$ & $\begin{array}{c}5.66 \\
\pm 0.71\end{array}$ & $"$ & $\begin{array}{r}79 \\
\pm 10\end{array}$ & $\begin{array}{r}11.5 \\
\pm 4.7\end{array}$ & van Leeuwen (2007) \\
\hline 10075 & 80725 & 7.02 & 0.2 & $\mathrm{~K} 2 \mathrm{~V}$ & 53.3 & $\begin{array}{l}51.20 \\
\pm 1.49\end{array}$ & 2.45 & $\begin{array}{r}43.9 \\
\pm 1.6\end{array}$ & $\begin{array}{r}1.6 \\
\pm 0.2\end{array}$ & ESA (1997) \\
\hline$"$ & $"$ & $"$ & $"$ & $"$ & $"$ & $\begin{array}{l}50.87 \\
\pm 0.80\end{array}$ & $"$ & $\begin{array}{r}44.1 \\
\pm 1.1\end{array}$ & $\begin{array}{r}1.6 \\
\pm 0.1\end{array}$ & van Leeuwen (2007) \\
\hline 12447 & 95589 & 7.44 & 0.2 & $\mathrm{~F} 8 \mathrm{~V}$ & 15.5 & $\begin{array}{l}13.96 \\
\pm 1.78\end{array}$ & 1.87 & $\begin{array}{l}134 \\
\pm 17^{(1)}\end{array}$ & $\begin{array}{c}3.1 \\
\pm 1.2^{(1)}\end{array}$ & ESA (1997) \\
\hline$"$ & $"$ & $"$ & $"$ & $"$ & $"$ & $\begin{array}{l}15.50 \\
\pm 1.27\end{array}$ & $"$ & $\begin{array}{l}121 \\
\pm 10^{(1)}\end{array}$ & $\begin{array}{c}2.3 \\
\pm 0.6^{(1)}\end{array}$ & van Leeuwen (2007) \\
\hline
\end{tabular}

(1) lower estimate of the error, using the parallax error only, and neglecting all the other (unknown) errors.

Although the two components of spectra G0V and K7V have a large magnitude difference: $\Delta m_{V} \approx 3.8 \mathrm{mag}$, the separation of the components is sufficiently large to allow the system to be observed photographically, for example by the multiple-exposure method with an objective grating (Hertzsprung, 1915). That method is particularly well suited to measuring wide components with a large magnitude difference. The quality of the photographic measurements made by long focal instruments between 1914 and 1983 is very good. Unfortunately since the end of this program, the quality has decreased significantly, and most of the available mea- surements come now from amateur astronomers, using small telescopes with a short focal length.

More than twenty orbits have been computed up to now. The first one was done by Powell (1861), and the latest by Strand (1969), was "computed with special attention to multiple-exposure photographic observations from 1914 to 1968 ". More than 45 years of new observations are now at our disposal, corresponding to 247 measures, including many photographic measures with a very good accuracy. We thus decided to compute new orbital elements, that are displayed in Table 4. All the available measurements were corrected for preces- 
sion, proper motion (see Fletcher, 1931a) and for radial velocity effects (see Fletcher, 1931b). The large number of available observations allowed us to applied rather strict selection criteria, and we rejected all the observations with residuals larger than 2 sigma (i.e., $2^{\circ} .2$ for $\theta$ and $0^{\prime \prime} .36$ for $\rho$ ). The new orbital elements are well determined, with small uncertainties (see Table 4). The period has the largest uncertainty, which is unavoidable, since only half of the orbit has been monitored yet (see Fig. 7). The fit is very good with mean residuals of $0^{\prime \prime} .123$ and $0^{\circ} .74$ for $\rho$ and $\theta$, respectively.

The values derived from this orbit are reported in Table 12. The parallax measured by Hipparcos leads to a total mass of $1.60 \pm 0.06 \mathrm{M}_{\odot}$, which is in very good agreement with the theoretical values of 1.07 and $0.55 \mathrm{M}_{\odot}$ for G0V and $\mathrm{K} 7 \mathrm{~V}$, respectively (Straizys and Kuriliene, 1981). The dynamical parallax is $0^{\prime \prime} .170$, very close to the Hipparcos measurement of $0^{\prime \prime} .168$.

\section{WDS 02020+0246 - STF 202 - ADS 1615 - HIP 9487 ( $\alpha$ Psc)}

This couple was discovered by W. Herschel on Oct. 19th 1779, with his 7-inch Newtonian telescope that he also used for discovering ADS 671 (Herschel, 1782, page 125). In a paper published in the Philosophical Transactions about the first observations of this object, J. Herschel et J. South reported "...this star has undergone no appreciable change..." (Herschel \& South, 1824, page 47). A clear motion was put to evidence for the first time much later by Secchi (1861, page 47 ) and Dawes (1867, page 310 ). The observations made in the following decades established that this motion is orbital. However, the motion has been very slow until now. The orbital period seems to be very large.

The observations are very numerous, but their quality is poor, especially before 1940 . Only 2 orbits have been computed until now, by Rabe (1961) and by Scardia (1983). The later begins to show systematic errors on the residuals, both for $\rho$ and $\theta$. In particular, the most recent observations indicate that the period is larger than what was previously assumed.

Taking advantage of 32 years of additional observations, we have revised the orbit using Kowalsky (1873)'s method, and rejecting all the aberrant observations. Although provisional, the orbital elements that we obtained (see Table 4) fit well the observations, with mean residuals of $0^{\prime \prime} .105$ and $1^{\circ} .24$ for $\rho$ and $\theta$, respectively.

The two stars of this couple were classified as A0pSiSr and A3m (Gray and Garrison, 1989, Hoffleit and Jaschek, 1991). The total mass obtained with Hipparcos parallax from van Leeuwen $(2007)$ is $3.7 \mathrm{M}_{\odot}$, in good agreement with theoretical values (i.e., 2.0 $\mathrm{M}_{\odot}$ for $\mathrm{A}$ stars). The dynamical parallax obtained with our elements and our formula of Scardia et al. (2008b) is $0^{\prime \prime} .020$ which is also in fair agreement with van Leeuwen's value of $0^{\prime \prime} .022$.

\section{WDS $02140+4729$ - STF 228 - ADS 1709 - HIP 10403}

This couple was discovered by F.G.W. Struve in Dorpat (now Tartu, Estonia) during the binary star survey he made in 1825-1827 with the famous $25-\mathrm{cm}$ refractor built by Fraunhofer (Struve, 1827). The first measurement was made in February 1829 and this couple has been then regularly monitored by the observers, even near the periastron passage, when the angular separation was about $0^{\prime \prime} .3$.

Many orbits have been computed, from the first published by Gore (1889) and the last by Söderhjelm (1999). Our orbit (Scardia, 1981) is still valid, but leads to systematic residuals in separation. A few tens of new measurements, mostly from interferometry, had been published since 1999, so we decided to do a new calculation. The big number of measurements allowed us to reject the observations leading to the largest residuals $\left(6^{\circ} .0\right.$ for $\theta$ and $0^{\prime \prime} .20$ for $\left.\rho\right)$. The companion has now accomplished more that one revolution since its discovery, and the orbital elements have a small uncertainty and can be considered as definitive.

The spectral of ADS 1709, is reported as F4V in SIMBAD, and as F2V+F7V in the WDS Catalog, which was determined by ten Brummelaar et al. (2000), from photometric measurements with the Hooker $2.5 \mathrm{~m}$ telescope of Mt.Wilson. The systemic mass with the Hipparcos parallax revised by van Leeuwen (2007), is $2.03 \pm 0.22 \mathrm{M}_{\odot}$, and $2.35 \pm 0.33 \mathrm{M}_{\odot}$ with the ESA (1997) original value. The latter is consistent with the value of $2.48 \mathrm{M}_{\odot}$ that is expected for a system F2V+F7V according to Straizys and Kuriliene (1981), and in good agreement with the values published by ten Brummelaar et al. (2000) and Malkov et al. (2012).

\section{WDS 06474+1812 - STT 156 - ADS 5447 - HIP 32539}

This couple was discovered by O Struve with the 38cm Merz-Mahler Poulkova refractor, during his survey aiming at founding new double stars (August 1841 December 1842) (Struve, 1843). Despite the small separation (about $0^{\prime \prime} .4$ ) and the difference of luminosity (1.4 mag.) between the two components, this discovery was doubtless for O. Struve who put the comment "certe oblonga" (certainly elongated) for this star. The first measurement was made in Dorpat by J.H. Madler on March 23rd 1843 with the famous $25 \mathrm{~cm}$ refractor built by Fraunhofer (Madler, 1845). This couple has been regularly observed since. The companion is now approaching the periastron that it should reach in 2017. The observations are becoming difficult with small instruments, since the separation is currently smaller than $0^{\prime \prime} .2$. Most of the published measurements over the last twenty years have been made by PISCO at Pic du Midi or in Merate.

The first orbit was computed by Dommanget (1953). The last one of Scardia et al. (2005) no longer 
represent the last observations. We thus revised our orbit of 2005, and computed new elements with the least squares Hellerich (1925)'s method. Unlike the last time, we obtained convergence and we can therefore give the uncertainties on the orbital elements. As usual, we have rejected all the aberrant observations, that had residuals larger than $7^{\circ} .0$ in $\theta$ and $0^{\prime \prime} .20$ in $\rho$.

The Hipparcos parallax values given by ESA (1997) and by van Leeuwen (2007) are $0^{\prime \prime} .00756$ and $0^{\prime \prime} .00566$, which is significantly different. As a consequence, our estimates of the total mass derived from those values are also very different: $4.84 \pm 2.80 \mathrm{M}_{\odot}(\mathrm{ESA})$ and $11.53 \pm 4.66 \mathrm{M}_{\odot}(\operatorname{van}$ Leeuwen, 2007). The dynamical parallax is $0^{\prime \prime} .0080$ is in good agreement with the ESA value of the Hipparcos parallax. The dynamical mass is $4.55 \mathrm{M}_{\odot}$.

The spectral type is reported as $\mathrm{A} 2 \mathrm{~V}$ in SIMBAD, which would lead to a theoretical total mass of $4.18 \mathrm{M}_{\odot}$ according to Straizys and Kuriliene (1981). We thus obtain a good agreement with our determination when we use the ESA parallax value. Recently Malkov et al. (2012) have computed for this couple three different values of the total mass, according to the method they used: $8.51 \mathrm{M}_{\odot}$ (dynamic), $5.18 \mathrm{M}_{\odot}$ (photometric) and $2.09 \mathrm{M}_{\odot}$ (spectroscopic).

\section{WDS 16289+1825 - STF 2052 - ADS 10075 - HIP 80725}

F.G.W. Struve observed this object for the first time in Dorpat (Estonia), with the Troughton meridian refractor $(\mathrm{D}=95 \mathrm{~mm})$, that was fitted with a filar micrometer built by Fraunhofer. He then repeated those observations during his big survey made in 1825-1827 with the $25 \mathrm{~cm}$ Dorpat refractor, and reported this pair as the number 2052 in his list. More historical information about this couple can be found in Scardia (1984).

Except when the companion is near the periastron, where $\rho \approx 0^{\prime \prime} .2$, this binary star is an easy target. It has been regularly observed since its discovery, and the measurements are plenty. The first orbit was computed by Jackson (1921) and the last by Lampens and Strigachev (2001). ADS 10075 belongs to the list of the targets we regularly observe with PISCO, and we decided to revise the elements of the first orbit published more than 30 years ago (Scardia, 1984). The orbital elements presented in Table 4 have small uncertainties and can be considered as definitive. The companion has nearly completed its first revolution since its discovery (see Fig. 7).

The sum of the masses, derived from the Hipparcos parallax is $1.62 \pm 0.14 \mathrm{M}_{\odot}$, in good agreement with the theory. The dynamical parallax is also in good agreement with the Hipparcos value (see Table 12). When comparing our orbit of 1984 (408 observations) and our new determination (537 obs.) the uncertainties on the systemic mass have decreased from 0.42 to $0.14 \mathrm{M}_{\odot}$.

\section{WDS 19266+2719 - STF 2525 - ADS 12447 - HIP 95589}

F.G.W. Struve discovered this binary star on Sept. 16th 1828, during his survey made in 1825-1827 with the 25-cm Dorpat refractor (Struve 1827). This pair is not a difficult target for observers except when the companion is close to its periastron, where its angular separation goes down to about $0^{\prime \prime} .1$. It has been regularly observed since its discovery and the observations are very numerous. However, because of the large eccentricity of the orbit, there is a lack of measurements around 1890 .

The first orbit (very preliminary) was published by Gore (1892) and the last one by Heintz (1984). Although the latter is still valid, we have decided to compute a new orbit to take advantage of the 128 new measurements of good quality that have been published since 1984. We have followed our usual procedure, but did not obtained convergence of the Hellerich's least squares method, which explains why the uncertainties are not displayed in Table 4 . Our orbit fit very well the observations with mean residuals of $0^{\prime \prime} .073$ for $\rho$ and $1^{\circ} .92$ for $\theta$.

The total mass derived from our elements and the Hipparcos parallax (van Leeuwen, 2007), is $2.3 \mathrm{M}_{\odot}$, in good agreement with the theory (see Strazys and Kuriliene, 1981). The dynamical parallax is $0^{\prime \prime} .0155$, which is in very good agreement with that value of the Hipparcos parallax (see Table 12).

\section{Conclusion}

We have presented here the 224 new measurements of 218 visual binaries that we have obtained with PISCO in 2014. The average accuracy was $0^{\prime \prime} .015$ for the angular separation and $0^{\circ} .5$ for the position angles. We have completed this data with the results of a study of the multiple system ADS 6993 with PISCO during the period 2004-2014 and proposed a new method to resolve part of the 180-degree ambiguity with the autocorrelations only. The AB component (SP 1) of this system was discovered at the Osservatorio Astronomico di Brera in 1888 by Schiaparelli. About 120 years later and still at the same observatory, we have been able to resolve it and obtain measurements of some additional components with a modern and more efficient technique and a bigger telescope.

We also presented new orbital elements computed for ADS 671, 1615, 1709, 5447, 10075 and 12447, that were partly derived from PISCO observations. The total mass values we have obtained are compatible with the expected theoretical values. The dynamical parallaxes computed with our revised formulae presented in Scardia et al. (2008b) are in very good agreement with Hipparcos values. 
The total number of measurements made with PISCO in Merate since its installation in 2004 now exceeds 3500. Our group has thus provided a good contribution to the continuing monitoring of long period visual binary systems, which is important for refining systemic stellar masses.

Acknowledgements. We thank the members of the U.S. Naval Observatory, Washington DC, for kindly sending us some lists of measurements of visual binaries. This work has made use of the Washington Double Star Catalog (http://ad.usno.navy.mil/wds/wds), the "Fourth Catalog of Interferometric Measurements of Binary Stars" (http://ad.usno.navy.mil/wds/int4), and the "Sixth Catalog of Orbits of Visual Binary Stars" (http://ad.usno.navy.mil/wds/orb6), maintained at the U.S. Naval Observatory. We also used the SIMBAD astronomical data base (http://simbad.u-strasbg.fr/simbad) operated by the Centre de Données Astronomiques de Strasbourg (France).

\section{References}

Aitken, R.G., 1932, "New General Catalog of Double Stars", Carnegie Institute, Washington

Aristidi, E., Carbillet, M., Lyon, J.-F., Aime, C., 1997, "Imaging binary stars by the cross-correlation technique", A\&AS, 125, 139

Baize, P., Romani, L., 1946, "Formules nouvelles pour le calcul des parallaxes dynamiques des couples orbitaux", Ann. Astrophys. 9, 13

Crossley, E., Gledhill, J., Wilson, J.M., 1879, " A Handbook of Double Stars", Macmillan \& Co., London

Dawes, R.W., 1867, "Catalogue of Micrometrical Measurements of Double Stars", Mem. R.A.S. 35, 137

Dommanget, J., 1953, Bull. R. Astron. Obs. Belgium 4, 150

ESA: 1997, The Hipparcos and Tycho Catalogues, ESA SP1200, ESA Publications Division, Noordwijk

Fletcher, A., 1931a, "Note on the effect of proper motion on double star measures", MNRAS, 92, 119

Fletcher, A., 1931b, "The binary system 61 Cygni", MNRAS, 92, 121

Gore, J.E., 1889, "On the orbit of Struve 228", MNRAS 50,81

Gore, J.E., 1892, "On the Orbit of S 2525”, MNRAS 53, 44

Gray, R.O., Garrison, R.F., 1989, "The late A-type stars - Refined MK classification, confrontation with Stromgren photometry, and the effects of rotation", AJ. Suppl. Ser. 70, 623

Hartkopf, W.I., Mason, B.D., 2016, "Sixth Catalog of Orbits of Visual Binary Stars" http://ad.usno.navy.mil/wds/orb6.html (OC6)

Hartkopf, W.I., Mason, B.D., Wycoff, G.L., McAlister, H.A., 2016, "Fourth Catalog of Interferometric Measurements of Binary Stars" http://ad.usno.navy.mil/wds/int4.html (IC4)

Heintz, W.D., 1984, "Orbits of 15 visual binaries", A\&A Suppl.Ser., 56, 5

Hellerich, J., 1925, "Uber eine Vereinfachung der Formeln der Bahnverbesserung visueller Doppelsterne", Astron. Nach., 223, 335
Herschel, W., 1782, "Catalogue of Double Stars", Phil. Trans. R. Soc. London 72, 112

Herschel, W., 1804, "Continuation of an Account of the Changes That Have Happened in the Relative Situation of Double Stars", Phil. Trans. R. Soc. London 94, 353

Herschel, J., South, J., 1824, "Observations of the Apparent Distances and Positions of 380 Double and Triple Stars, Made in the Years 1821, 1822, and 1823, and Compared with Those of Other Astronomers; Together with an Account of Such Changes as Appear to Have Taken Place in Them Since Their First Discovery. Also a Description of a Five-Feet Equatorial Instrument Employed in the Observations", Phil. Trans. R. Soc. London 114, 1

Hertzsprung, E., 1915, "Photographische Beobachtungen einiger hellen Doppelsterne", Astron. Nach., 200, 105

Hoffleit, D., Jaschek, C., 1991, "The Bright Star Catalog", Yale University Obs., 5th rev. ed

Jackson, J., 1921, Greenwich Double Star Cat., 213

Kowalsky, M., 1873, Procès-verbaux de l'Université Impériale de Kasan

Lampens, P., Strigachev, A., 2001, "Multicolour observations of nearby visual double stars. New CCD measurements and orbits", A\&A, 368, 572

Madler, J.H., 1845, Beobach. Univ. Sternw. Dorpat 11, 49

Malkov, O.Yu., Tamazian, V.S., Docobo, J.A., Chulkov, D.A., 2012, "Dynamical masses of a selected sample of orbital binaries", A\&A 546, 69

Mason, B.D., Wycoff, G.L., Hartkopf, W.I., 2016, "Washington Double Star Catalog" http://ad.usno.navy.mil/wds/wds.html (WDS)

Powell, E.B., 1861, "On the Binary Star eta Cassiopeae", MNRAS 21, 65

Prieur, J.-L, Koechlin, L., André, C., Gallou, G., Lucuix, C., 1998, "The PISCO speckle camera at Pic du Midi Observatory", Experimental Astronomy, vol 8, Issue 4, 297

Prieur, J.-L., Scardia, M., Pansecchi, L., Argyle, R.W., Sala, M., Ghigo, M., Koechlin, L., Aristidi, E., 2008, "Speckle observations with PISCO in Merate. V. Astrometric measurements of visual binaries in 2006", MNRAS, 387, 772 (Paper V)

Prieur, J.-L., Scardia, M., Pansecchi, L., Argyle, R.W., Sala, M., 2009, "Speckle observations with PISCO in Merate. VII. Astrometric measurements of visual binaries in 2007", MNRAS, 395, 907 (Paper VII)

Prieur, J.-L., Scardia, M., Pansecchi, L., Argyle, R.W., Sala, M., 2010, "Speckle observations with PISCO in Merate. VIII. Astrometric measurements of visual binaries in 2008", MNRAS, 407, 1913 (Paper IX)

Prieur, J.-L., Scardia, M., Pansecchi, L., Argyle, R.W., Sala, M., 2012, "Speckle observations with PISCO in Merate. XI. Astrometric measurements of visual binaries in 2010", MNRAS, 422, 1057 (Paper XI)

Prieur, J.-L., Scardia, M., Pansecchi, L., Argyle, R.W., Zanutta, A., Aristidi, E., 2014, "Speckle observations with PISCO in Merate (Italy). XIII. Astrometric measurements of visual binaries in 2012 and new orbits for ADS 10786 BC, 12144, 12515, 16314 and 16539", Astron. Nach., 335, 817 (Paper XIII)

Rabe, W., 1961, "Doppelsternbahnen von Wilhelm RABE", Veroff. Sternw. Munchen 6, 4, 113 
Scardia, M., 1983, "Elements orbitaux de cinq étoiles doubles visuelles", Astron. Nach., 304, 5, 257

Scardia, M., 1984, "Elements orbitaux, presque définitifs, de l'étoile double visuelle ADS 10075 - STF 2052", Astron. Nach., 305, 3, 127

Scardia, M., Prieur, J.-L., Sala, M., Ghigo, M., Koechlin, L., Aristidi, E., Mazzoleni, F., 2005, "Speckle observations with PISCO in Merate. I. Astrometric measurements of visual binaries in 2004", MNRAS, 357, 1255 (with erratum in MNRAS 362, 1120) (Paper I)

Scardia, M., Prieur, J.-L., Pansecchi, L., Argyle, R.W., Sala, M., Ghigo, M., Koechlin, L., Aristidi, E., 2006, "Speckle observations with PISCO in Merate. II. Astrometric measurements of visual binaries in 2004", MNRAS, 367, 1170 (Paper II)

Scardia, M., Prieur, J.-L., Pansecchi, L., Argyle, R.W., Basso, S., Sala, M., Ghigo, M., Koechlin, L., Aristidi, E., 2007, "Speckle observations with PISCO in Merate. III. Astrometric measurements of visual binaries in 2005 and scale calibration with a grating mask", MNRAS, 374, 965 (Paper III)

Scardia, M., Prieur, J.-L., Pansecchi, L., Argyle, R.W., Sala, M., Basso, S., Ghigo, M., Koechlin, L., Aristidi, E., 2008a, "Speckle observations with PISCO in Merate. IV. Astrometric measurements of visual binaries in 2005", Astron. Nach., 329, 1, 54 (Paper IV)

Scardia, M., Prieur, J.-L., Pansecchi, L., Argyle, R.W., 2008b, "Preliminary orbital elements of six visual binary stars and revised version of the Baize-Romani dynamical parallax", Astron. Nach., 329, 379

Scardia M., Prieur J.-L., Pansecchi L., Argyle R.W., Sala M., 2009, "Speckle observations with PISCO in Merate. VI. Astrometric measurements of visual binaries in 2006", Astron. Nach., 330, 1, 55 (Paper VI)

"Speckle observations with PISCO in Merate. VIII. Astrometric measurements of visual binaries in 2007 and new orbits of the multiple system Zeta Aqr",

Scardia, M., Prieur, J.-L., Pansecchi, L., Argyle, R.W., Sala, M., 2010, Astron. Nach., 331, 286 (Paper VIII)

Scardia, M., Prieur, J.-L., Pansecchi, L., Argyle, R.W., Sala, M., 2011, "Speckle observations with PISCO in Merate. X. Astrometric measurements of visual binaries in 2009", Astron. Nach., 332, 508 (Paper X)

Scardia, M., Prieur, J.-L., Pansecchi, L., Argyle, R.W., Spanó, P., Riva, M., Landoni, M., 2013, "Speckle observations with PISCO in Merate. XII. Astrometric measurements of visual binaries in 2011", MNRAS, 434, 2803 (Paper XII)

Scardia, M., Prieur, J.L., Pansecchi, L., Argyle, R.W., Zanutta, A., Aristidi, E., 2015a, "Speckle observations with PISCO in Merate (Italy). XIV. Astrometric measurements of visual binaries in 2013 and new orbits for ADS 1097, 5871, 7203, 7775, 9378, 9578 and 11186", Astron. Nachr., 336, 388 (Paper XIV)

Scardia, M., Prieur, J.L., Pansecchi, L., Argyle, R.W., Zanutta, 2015b, IAU Commission 26, Inf. circ. 185

Scardia, M., Prieur, J.L., Pansecchi, L., Argyle, R.W., Zanutta, 2015c, IAU Commission 26, Inf. circ. 186

Schiaparelli, G.V., 1909, "Osservazioni sulle stelle doppie. Serie seconda: comprendente le misure di 636 sistemi eseguite col refrattore equatoriale Merz-Repsold negli anni 1886-1900", Pubblicazioni del Reale Osservatorio
Astronomico di Brera vol. 46

Secchi, A., 1860, "Catalogo di 1321 stelle doppie misurate col grande equatoriale di Merz all'osservatorio del Collegio Romano e confrontate con le misure anteriori", Tipografia delle Belle Arti, Roma

Söderhjelm, S., 1999, "Visual binary orbits and masses post Hipparcos", A\&A 341, 121

Straizys, V., Kuriliene, G., 1981, "Fundamental stellar parameters derived from the evolutionary tracks", AP\&SS, 80, 353. Astrophysics and Space Science

Strand, K.A., 1969, "The orbit of Eta Cassiopeiae", AJ 74, 760

Struve, F.G.W., 1827, "Catalogus novus stellarum duplicium et multiplicium maxima ex parte in specula Universitatis Caesareae Dorpatensis per magnum telescopium achromaticum Fraunhoferi detectarum", Dorpat, Schuenmann Ed.

Struve, O., 1843, "Catalogue de 514 étoiles doubles et multiples", Académie Impériale des Sciences, St. Petersbourg

ten Brummelaar, T. et al., 2000, "Binary Star Differential Photometry Using the Adaptive Optics System at Mount Wilson Observatory", AJ. 119, 2403

van Leeuwen, F., 2007, "Hipparcos, the new reduction of the raw data", Springer Netherlands Ed. 